\title{
ON THE SATO-TATE CONJECTURE FOR NON-GENERIC ABELIAN SURFACES
}

\author{
CHRISTIAN JOHANSSON, WITH AN APPENDIX BY FRANCESC FITÉ
}

ABSTRACT. We prove many non-generic cases of the Sato-Tate conjecture for abelian surfaces as formulated by Fité, Kedlaya, Rotger and Sutherland, using the potential automorphy theorems of Barnet-Lamb, Gee, Geraghty and Taylor.

\section{INTRODUCTION}

Let $E$ be an elliptic curve over a number field $F$ without complex multiplication $(\mathrm{CM})$ and let $v$ be a finite prime of $F$ with residue field $\mathbb{F}_{q_{v}}$ such that $E$ has good reduction at $v$. Hasse's Theorem tells us that the number of $\mathbb{F}_{q_{v}}$-points of $E$ is $q_{v}+1-a_{v}$, where $a_{v}$ is an integer such that $\left|a_{v}\right| \leq 2 q_{v}^{1 / 2}$. The Sato-Tate conjecture concerns the distribution of the numbers $a_{v} / 2 q_{v}^{1 / 2}$ in $[-1,1]$ as $v$ varies through the primes of good reduction for $E$. More precisely, it tells us that the numbers $\theta_{v}=\arccos \left(a_{v} / 2 q_{v}^{1 / 2}\right)$ are distributed according to the measure $\frac{2}{\pi} \sin ^{2} \theta d \theta$ on $[0, \pi]$. This conjecture was recently settled in [HSBT and BGHT] (see also [BLGG] when $F$ is totally real as a result of progress on potential automorphy theorems for compatible systems of Galois representation of arbitrary dimension. When $E$ has CM over $F$, the $\theta_{v}$ are uniformly distributed in $[0, \pi]$; when $E$ has CM but not over $F$ the $\theta_{v}$ are equidistributed on $[0, \pi]$ according to the measure which is half of the uniform probability measure over $[0, \pi]$ plus a Dirac measure of mass $1 / 2$ at $\pi / 2$.

All known proofs follow a pattern similar to that of the classical proof of the Prime Number Theorem and its generalizations, where holomorphy and nonvanishing of certain (complex) $L$-functions are established in the region $R e s \geq 1$. In the CM cases, these $L$-functions are understood in terms of Hecke $L$-functions. In the non-CM case, Tate realized that the same proof would work if one could establish the required properties for the symmetric power $L$-functions of the elliptic curve. These observations were generalized and axiomatized by Serre ([Ser1, Appendix to $\$ 1]$ ). It was then observed by Langlands that the required analytic properties follow from the automorphy of associated $L$-functions, and this opened up possibilities both in terms of proofs and further generalizations. During these developments, the Sato-Tate conjecture was also reinterpreted as a statement about the equidistribution of a certain sequence of elements (the images of Frobenii, suitably normalized) inside a certain compact Lie group (that turns out to be $\mathrm{SU}(2)$ for the original Sato-Tate conjecture). If one believes that all motivic $L$-functions are automorphic, then the Sato-Tate conjecture becomes a statement about the distribution of the Satake parameters of unitary automorphic representations.

Received by the editors May 19, 2014 and, in revised form, September 22, 2015.

2010 Mathematics Subject Classification. Primary 11F80, 11G10. 
In [FKRS], Fité, Kedlaya, Rotger and Sutherland described the generalization of the Sato-Tate conjecture to abelian surfaces. Before we describe their results, let us take a step back and sketch how a fully functional Langlands philosophy would apply to the situation of abelian surfaces (the same type of argument should apply to any "pure motive"). Let $A / F$ be an abelian surface. Then the Langlands correspondence predicts the existence of an $L$-algebraic automorphic representation $\pi_{A}$ attached to the compatible system $\left(H_{e t}^{1}\left(A, \overline{\mathbb{Q}}_{\ell}\right)\right)_{\ell}$ and associated with $\pi_{A}$ is a representation $\rho_{A}: L_{F} \rightarrow \mathrm{GL}_{4}(\mathbb{C})$ of the conjectural Langlands group $L_{F}$. We define the Sato-Tate group of $A$ to be the image $G=\left(\rho_{A} \otimes|\cdot|^{1 / 2}\right)\left(L_{F}\right)$ where $|\cdot|$ denotes the norm character of $L_{F}$. The representation $\rho_{A} \otimes|\cdot|^{1 / 2}$ is unitary and the group $G$ should be a compact group. The Sato-Tate conjecture would then say that the sequence of conjugacy classes $\left(\left(\left.\rho_{A} \otimes|\cdot|\right|^{1 / 2}\right)\left(F r o b_{v}\right)\right)_{v \notin S}$ (where $S$ is the set of finite primes where $A$ has bad reduction) is equidistributed with respect to the measure on the set of conjugacy classes of $G$ induced from the Haar probability measure on $G$. Following the strategy of Serre, this follows from the holomorphy and non-vanishing in the region $R e s \geq 1$ of the partial $L$-functions $L^{S}\left(s, r \circ\left(\rho_{A} \otimes|\cdot|^{1 / 2}\right)\right)$ for all non-trivial irreducible representations $r$ of $G$. The global Langlands correspondences would then imply that each $L^{S}\left(s, r \circ\left(\rho_{A} \otimes|\cdot|{ }^{1 / 2}\right)\right)$ is the partial $L$-function of a unitary cuspidal automorphic representation of $\mathrm{GL}_{\operatorname{dim} r}\left(\mathbb{A}_{F}\right)$ (which is not of form $|\cdot|^{i \lambda}, \lambda \in \mathbb{R}$ ) and this gives the holomorphy and non-vanishing. All this is of course highly conjectural and the existence of $L_{F}$ is particularly problematic. Instead, in order to get something well-defined, one has to use the compatible system $\left(H_{e t}^{1}\left(A, \overline{\mathbb{Q}}_{\ell}\right)\right)_{\ell}$ directly; this is a construction due to Serre ([Ser2, §8]). This gives a substitute (denoted $S T_{A}$ ) for $G$ as well as the conjugacy classes $\left(\left(\rho_{A} \otimes|\cdot|^{1 / 2}\right)\left(F r o b_{v}\right)\right)_{v \notin S}$, and Serre formulates the Sato-Tate conjecture in this generality. For abelian surfaces, Fité, Kedlaya, Rotger and Sutherland (in [FKRS] give a precise conjecture of what $S T_{A}$ should be; they obtain 52 possibilities, each given by an explicit recipe in terms of the endomorphisms of $A$.

In this paper we wish to investigate what can be said about the Sato-Tate conjecture for abelian surfaces using current potential automorphy theorems. Roughly speaking there are three cases. First, there is the so-called generic case when $\operatorname{End}_{\overline{\mathbb{Q}}}(A)=\mathbb{Z}$. In this case we are not able to prove anything because of current restrictions on potential automorphy theorems to Galois representations that are regular. Second, we have the cases when $H_{e t}^{1}\left(A, \overline{\mathbb{Q}}_{\ell}\right)$ is potentially abelian where one may prove the conjecture completely, using class field theory. The remaining cases may be loosely described as those that are potentially of $\mathrm{GL}_{2}$-type (see Definition 18 for our slightly non-standard definition of $\mathrm{GL}_{2}$-type) but not potentially abelian; here we may prove the conjecture under the restriction that a certain (at most quadratic) extension of $F$ is totally real. Unsurprisingly, the situation and required assumptions mirror that of the elliptic curve case. The proofs are somewhat more delicate due to the more complicated structure of $S T_{A}$ but otherwise follow the general strategy as in [HSBT]. The results we prove may be summarized loosely as follows.

Theorem 1. Let $A / F$ be an abelian surface.

1) (Propositions 22, 24, 26, 27 and 29) If $A$ is of type $\boldsymbol{B}, \boldsymbol{C}$ or $\boldsymbol{E}$ and a certain at most quadratic extension of $F$ is totally real, then the Sato-Tate conjecture holds for $A$. 
2) (Proposition [16) If $A$ is of type $\boldsymbol{D}$ or $\boldsymbol{F}$, then the Sato-Tate conjecture holds for $A$.

Here type $\mathbf{B}, \mathbf{C}, \mathbf{D}, \mathbf{E}$ resp. $\mathbf{F}$ refers to the (absolute) type as defined in FKRS, $\S 4]$; we recall them in section 2 . For now, it suffices to say that $\mathbf{D}$ and $\mathbf{F}$ are the potentially abelian cases, and that the excluded type $\mathbf{A}$ is the generic case. Special cases of Theorem 1 that have previously been recorded in the literature are the case when $A / F$ is isogenous to a product of non-CM elliptic curves that do not become isogenous over any finite extension and $F$ is totally real ([Har $)$, and a number of cases of type $\mathbf{F}$ over $\mathbb{Q}$ ([FS]).

Let us outline the contents of the paper. Section 2 states the conjecture, explains the strategy and sets up notation and terminology to be used throughout the paper. The reader is advised to read this before proceeding, or look back at it later if unfamiliar notation is encountered. In section 3 we do the potentially abelian cases. Indeed, we give a general equidistribution result for continuous unitary representations $W_{F} \rightarrow \mathrm{GL}_{n}(\mathbb{C})$, roughly following the "ideal" strategy above. This is certainly well known but we could not find a precise reference, so we have included some details. For abelian varieties $A$ whose Tate module is potentially abelian this gives a "Sato-Tate conjecture" for $A$, but it is not clear that the group obtained in this fashion agrees with the one defined by Serre. We also prove the Sato-Tate conjecture in the form of Serre. In section 4 we do the cases potentially of $\mathrm{GL}_{2}{ }^{-}$ type, using the powerful potential automorphy results of [BLGGT]. We believe, and hope that the reader agrees, that the relatively clean proofs of section 4 are a good illustration of the power and beauty of the results of [BLGGT]. The paper concludes with an Appendix, written by Francesc Fité, giving examples of abelian surfaces satisfying the assumptions of Propositions 24 and 29, showing that they are non-empty.

\section{The CONJECTURE And The STRATEgy}

We will fix once and for all isomorphism $\iota: \overline{\mathbb{Q}}_{\ell} \cong \mathbb{C}$ for all $\ell$. (For simplicity we will omit $\ell$ from the notation; we believe this should not cause any confusion.) We will let $\overline{\mathbb{Q}}$ denote the algebraic closure of $\mathbb{Q}$ in $\mathbb{C}$. If $K$ is any number field, then, using $\iota$, we may identify embeddings $K \hookrightarrow \overline{\mathbb{Q}}_{\ell}$ and $K \hookrightarrow \overline{\mathbb{Q}}$.

2.1. The Sato-Tate group and the definition of conjugacy classes. In this subsection we recall [FKRS, §2.1]. Let $A$ be an abelian variety of dimension $g$ over a number field $F$, let $\phi$ be a polarization of $A$ and fix an embedding $F \hookrightarrow \overline{\mathbb{Q}}$. Fix a symplectic basis for $H_{1}\left(A(\mathbb{C})^{\text {an }}, \mathbb{Q}\right)$ and use it to equip that space with an action of $\operatorname{GSp}_{2 g}(\mathbb{Q})$. Fix a prime $\ell$ and let $V_{\ell}(A)$ be the rational $\ell$-adic Tate module of $A$. Make the identifications

$$
\begin{aligned}
V_{\ell}(A) & \cong H_{1, e t}\left(A_{\overline{\mathbb{Q}}_{\ell}}, \mathbb{Q}_{\ell}\right) \cong H_{1, e t}\left(A_{\mathbb{C}}, \mathbb{Q}_{\ell}\right) \\
& \cong H_{1}\left(A(\mathbb{C})^{a n}, \mathbb{Q}_{\ell}\right) \cong H_{1}\left(A(\mathbb{C})^{a n}, \mathbb{Q}\right) \otimes_{\mathbb{Q}} \mathbb{Q}_{\ell} .
\end{aligned}
$$

The Weil pairing becomes identified with the cup product pairings in étale and singular cohomology and our symplectic basis for $H_{1}\left(A(\mathbb{C})^{a n}, \mathbb{Q}\right)$ gives a symplectic basis for $V_{\ell}(A)$, and the action of $G_{F}=\operatorname{Gal}(\overline{\mathbb{Q}} / F)$ defines a continuous homomorphism

$$
\rho_{A, \ell}: G_{F} \rightarrow \operatorname{GSp}_{2 g}\left(\mathbb{Q}_{\ell}\right)
$$


We let $G_{\ell}=G_{\ell}(A)$ denote the image of $\rho_{A, \ell}$, and $G_{\ell}^{Z a r}=G_{\ell}^{Z a r}(A)$ denotes the Zariski closure of $G_{\ell}$ inside $\mathrm{GSp}_{2 g}\left(\mathbb{Q}_{\ell}\right)$ (the $\ell$-adic monodromy group of $A$ ). $G_{\ell}$ is open in $G_{\ell}^{Z a r}$ in the $\ell$-adic topology. Going further we let $G_{F}^{1}$ denote the kernel of the cyclotomic character $\chi_{\ell}: G_{F} \rightarrow \mathbb{Z}_{\ell}^{\times}$and set $G_{\ell}^{1}=G_{\ell}^{1}(A)$ to be the image of $G_{F}^{1}$ under $\rho_{A, \ell}$; we denote by $G_{\ell}^{1, Z a r}=G_{\ell}^{1, Z a r}(A)$ the Zariski closure of $G_{\ell}^{1, Z a r}$ in $\mathrm{GSp}_{2 g}\left(\mathbb{Q}_{\ell}\right) . G_{\ell}^{1, Z a r}$ coincides with the kernel of the similitude character on $G_{\ell}^{Z a r}$. If $F^{\prime} / F$ is a finite extension, then $G_{\ell}^{1, Z a r}\left(A_{F^{\prime}}\right)$ is a finite index subgroup of $G_{\ell}^{1, Z a r}(A)$, hence these groups have the same identity components. Moreover, for sufficiently large $F^{\prime}, G_{\ell}^{1, Z a r}\left(A_{F^{\prime}}\right)$ is connected.

Using $\iota$ we may embed $\operatorname{GSp}_{2 g}\left(\mathbb{Q}_{\ell}\right)$ into $\mathrm{GSp}_{2 g}(\mathbb{C})$. Put $G^{1}=G_{\ell}^{1, Z a r} \otimes_{\mathbb{Q}_{\ell}, \iota} \mathbb{C}$ and $G=G_{\ell}^{Z a r} \otimes_{\mathbb{Q}_{\ell}, \iota} \mathbb{C}$. Let $v$ be a finite prime of $F$ with residue field $\mathbb{F}_{q_{v}}$ (we will use this notation throughout the paper). We may identify $G / G^{1}$ with $\mathbb{C}^{\times}$compatibly with the cyclotomic character, and the image of $g_{v}=\rho_{A, \ell}\left(F r o b_{v}\right) \in G$ in $\mathbb{C}^{\times}$is $q_{v}$ (here and throughout the rest of this paper $F_{r o b}$ denotes the arithmetic Frobenius). An argument due to Deligne shows that $G_{\ell}^{Z a r}$ contains the central $\mathbb{G}_{m}$ of $\mathrm{GSp}_{2 g}$. Hence $g_{v}^{\prime}=q_{v}^{-1 / 2} g_{v} \in G^{1}$.

Definition 2. 1) The Sato-Tate group $S T_{A}$ of $A$ (for the prime $\ell$ and isomorphism $\iota)$ is a maximal compact Lie subgroup of $G^{1}$ contained in $\operatorname{USp}(2 g)$.

2) The semisimple component of $g_{v}^{\prime}$ is an element of $G^{1}$ with eigenvalues of norm 1 and hence belongs to some conjugate of $S T_{A}$. Thus we may define $s(v)$ to be the associated conjugacy class in $S T_{A}$.

Remark 3. We have phrased the definitions in the general form of [Ser2, §8]. In the case of abelian varieties, semisimplicity is well known and due to Tate. Moreover, $G_{\ell}^{Z a r}$, and hence $G_{\ell}^{1, Z a r}$ and $S T_{A}$, is reductive by Faltings's theorem.

We remark that when $g \leq 3$ or $A$ is isogenous to a product of CM abelian varieties, the $G_{\ell}^{1, Z a r}$ is known to have a common model over $\mathbb{Q}([\mathrm{FKRS}$, Theorem 2.16], [BK, Theorem 6.6]), so $S T_{A}$ is a compact real form of $G^{1}$ and independent of $\ell$. Note however that it is not in general known in these cases that the conjugacy classes $s_{v}$ are independent of $\ell$. We may now state the generalized Sato-Tate conjecture:

Conjecture 4 ([Ser2, §8], [FKRS, Conjecture 1.1]). The classes $s(v)$ are equidistributed in the set $\operatorname{Conj}\left(S T_{A}\right)$ of conjugacy classes of $S T_{A}$ with respect to the pullback of the Haar probability measure on $S T_{A}$ to $\operatorname{Conj}\left(S T_{A}\right)$.

2.2. The strategy of proof (following Tate and Serre). Let $S$ be a finite set of places of $F$ containing the infinite places such that $\rho_{A, \ell}$ is unramified outside $S$. Let $r$ be an irreducible representation of $S T_{A}$. We may form the formal product

$$
L^{S}(s, r, A)=\prod_{v \notin S} \frac{1}{\operatorname{det}\left(1-r(s(v)) q_{v}^{-s}\right)}
$$

where by abuse of notation we let $s(v)$ denote some element of the conjugacy class $s(v)$ (any element will $\operatorname{do} ; \operatorname{det}\left(1-r(s(v)) q_{v}^{-s}\right)$ is independent of the choice). Then Serre, elaborating on the special case $S T_{A}=\mathrm{SU}(2)$ which was studied by Tate, proves the following. 
Theorem 5 (Serre, [Ser1, Appendix to §1]). Assume that, for any irreducible representation $r, L^{S}(s, r, A)$ converges absolutely on $\operatorname{Re}(s)>1$ and extends to a meromorphic function on $\operatorname{Re}(s) \geq 1$ having no zeroes or poles except possibly at $s=1$. Then $(s(v))_{v \notin S}$ are equidistributed in $\operatorname{Conj}\left(S T_{A}\right)$ if and only if $L^{S}(s, r)$ is holomorphic and non-vanishing at $s=1$ for all irreducible $r \neq 1$.

In order to prove anything about the functions $L^{S}(s, r, A)$ we will have to identify them with partial $L$-functions of geometric Galois representations. Since the analytic properties we require only depend on knowing the Euler factors at all but finitely many places we will not worry too much about the distinction between the full $L$-function and its various incomplete/partial $L$-functions. This should hopefully not cause any confusion. The basic strategy, due to Taylor ([Tay]; see also [HSBT] ), for proving these sorts of analytic results is to combine potential automorphy results with Brauer's theorem and known analytic properties of various (complex analytic) $L$-functions. The former are currently restricted to regular Galois representations (i.e. with distinct Hodge-Tate weights); using the latter techniques one may enlarge the class of $L$-functions whose analytic behaviour can be studied to handle the cases required for Theorem 1 .

2.3. The cases. In FKRS, 52 cases of possible Sato-Tate groups are identified (35 of them possible over totally real fields, 34 over $\mathbb{Q}$ ), of which we will exclude the case $S T_{A}=\mathrm{USp}(4)$ (the generic case) as it seems to be intractable using current technology. The 52 cases correspond to 52 so-called "Galois types", giving the abstract structure of $\operatorname{End}_{L}(A) \otimes_{\mathbb{Z}} \mathbb{R}$ as a representation of $\operatorname{Gal}(L / F)$, where $L$ is the minimal extension of $F$ over which all endomorphisms of $A$ are defined. After excluding the generic case (Galois type $\mathbf{A}$ ), the remaining Galois types are divided into 5 "types" (or "absolute types") B, C, D, E and F. They correspond to the following arithmetic interpretations:

- B : $A_{L}$ is either isogenous to a product of non-isogenous elliptic curves without CM or simple with real multiplication.

- $\mathbf{C}: A_{L}$ is isogenous to a product of two elliptic curves, one with CM and the other one without.

- D : $A_{L}$ is either isogenous to a product of non-isogenous elliptic curves with CM or simple with CM by a quartic field.

- $\mathbf{E}: A_{L}$ is either isogenous to the square of an elliptic curve without $\mathrm{CM}$ or simple with QM (quaternionic multiplication).

- $\mathbf{F}: A_{L}$ is isogenous to the square of an elliptic curve with CM.

We will obtain complete results for cases $\mathbf{D}$ and $\mathbf{F}$ from a general study of potentially abelian, geometric Galois representations in section 3 and do a case-by-case study of types $\mathbf{B}, \mathbf{C}$ and $\mathbf{E}$ in section 4 ,

2.4. Some notation and terminology. When classifying the irreducible representations of the Sato-Tate groups we will use specific presentations of them as subgroups of USp(4), which we will give as a subgroup of $\mathrm{GL}_{4}(\mathbb{C}$ ) (using different alternating forms in different cases). To make this paper easier to read in conjunction with [FKRS, we have tried to stick with their notation (which can be found in $\S 3$ of their paper) as much as possible; the main difference is that we use $F$ to denote the field of definition of $A$ (they use $k$ ) and $L$ to denote the minimal field of definition of all endomorphisms (they use $K$; we will instead use $K$ to denote a quadratic field inside $\left.\operatorname{End}_{L}(A) \otimes_{\mathbb{Z}} \mathbb{Q}\right)$. We set $\operatorname{End}_{F^{\prime}}(A)^{0}=\operatorname{End}_{F^{\prime}}(A) \otimes_{\mathbb{Z}} \mathbb{Q}$ for 
any extension $F^{\prime} / F$. Next, let us recall the notation of [FKRS] for various matrices and subgroups of $\mathrm{GL}_{4}(\mathbb{C})$ here for the convenience of the reader, and make a few additions. Define

$$
J_{2}=\left(\begin{array}{cc}
0 & 1 \\
-1 & 0
\end{array}\right) \in \mathrm{GL}_{2}(\mathbb{C}), \quad J=\left(\begin{array}{cc}
0 & J_{2} \\
-J_{2} & 0
\end{array}\right) \in \mathrm{GL}_{4}(\mathbb{C})
$$

and

$$
S=\left(\begin{array}{cc}
0 & I d_{2} \\
-I d_{2} & 0
\end{array}\right), \quad S^{\prime}=\left(\begin{array}{cc}
J_{2} & 0 \\
0 & J_{2}
\end{array}\right)
$$

We use the standard presentations

$$
\begin{gathered}
\mathrm{U}(n)=\left\{A \in \mathrm{GL}_{n}(\mathbb{C}) \mid A^{*} A=I d_{n}\right\}, \\
\mathrm{SU}(n)=\{A \in \mathrm{U}(n) \mid \operatorname{det}(A)=1\}
\end{gathered}
$$

for the unitary and special unitary groups, where $A^{*}$ denotes the conjugate-transpose of $A$. For types $\mathbf{B}$ and $\mathbf{C}$ we will use the presentation

$$
\mathrm{USp}(4)=\left\{A \in \mathrm{U}(4) \mid A^{t} S^{\prime} A=S^{\prime}\right\} .
$$

For type $\mathbf{E}$ we use the presentation

$$
\mathrm{USp}(4)=\left\{A \in \mathrm{U}(4) \mid A^{t} S A=S\right\} .
$$

We record three matrices here that will be of use in later sections:

$$
a=\left(\begin{array}{cc}
J_{2} & \\
& I d_{2}
\end{array}\right), \quad b=\left(\begin{array}{cc}
I d_{2} & \\
& J_{2}
\end{array}\right), \quad c=\left(\begin{array}{cc}
I d_{2} \\
-I d_{2} &
\end{array}\right) .
$$

Note the slight inconsistency $c=S$; note also that $a b=S^{\prime}$. This follows the usage in [FKRS] (though they have no notation for what we are calling $S^{\prime}$ other than $a b$ ); $S$ and $S^{\prime}$ will only be used as matrices defining symplectic forms and $a, b, c$ and their products will only be used to describe elements of our Sato-Tate groups.

We will use the word character for what is sometimes called a quasicharacter, i.e., we do not require that characters are unitary unless specified. We will follow the usage in $\mathrm{Har}$ and say that a meromorphic function $f: U \rightarrow \mathbb{C}$ where $U \subseteq \mathbb{C}$ is open and contains $\{R e s \geq 1\}$ is invertible if it is holomorphic and non-vanishing on $\{\operatorname{Re} s \geq 1\}$. Following the usage in FKRS we will let $F_{r o b}$, for $v$ a finite place of a number field $F$, denote an arithmetic Frobenius element, and we will use the Tate module of our abelian surfaces (as opposed to their duals which we used in the introduction). In accordance with this choice we will normalize local class field theory by sending uniformizers to arithmetic Frobenii and attach Galois representation to automorphic representations of general linear groups by matching up Satake parameters with eigenvalues of arithmetic Frobenii.

\section{Equidistribution LAWS FOR POTENTIALLY ABELIAN GEOMETRIC Galois REPRESENTATIONS}

The aim of this section is to record equidistribution results for $\ell$-adic Galois representations that are geometric and potentially abelian. This is done by giving a natural correspondence between such Galois representation and Weil parameters (i.e. continuous complex representations of the global Weil group) that is characterized by the fact that it preserves trace of Frobenius at unramified places. For unitary Weil parameters satisfying a mild condition on the image one may prove an equidistribution result. Neither statements nor proofs in this section should surprise an expert, but we have not found a reference for some of the results that 
we present (the abelian case is done in [Ser1]). Some of the results in this section are covered in Tat and Wei] we have included a few details for the convenience of the reader.

3.1. Weil parameters and potentially abelian Galois representations. Let us start by defining the main objects of interest in this section. At the suggestion of the referee, we sketch the definition of the Weil group of a number field $F$, and we refer to AT] and Tat for the details. Given a finite Galois extension $E / F$, the relative Weil group $W_{E / F}$ is the extension

$$
1 \rightarrow C_{E} \rightarrow W_{E / F} \rightarrow \operatorname{Gal}(E / F) \rightarrow 1
$$

defined by the fundamental class $\alpha_{E / F} \in H^{2}(\operatorname{Gal}(E / F), C)$ of global class field theory, where $C_{E}=E^{\times} \backslash \mathbb{A}_{E}^{\times}$is the idele class group and $C=\lim _{E} C_{E}$ (direct limit taken over all finite $E / F)$. $W_{E / F}$ inherits a group topology from $C_{E}$ and the Weil group $W_{F}$ is the topological group $W_{F}=\lim _{E} W_{E / F}$.

Definition 6. 1) A Weil parameter is a continuous semisimple complex finitedimensional representation of $W_{F}$.

2) A Weil parameter $r: W_{F} \rightarrow \mathrm{GL}_{n}(\mathbb{C})$ is said to be algebraic if for any place $v \mid \infty$ of $F,\left.r\right|_{W_{\overline{F_{v}}}}$ is a direct sum of characters of the form $z \mapsto z^{p} \bar{z}^{q}$ (for some $p, q \in \mathbb{Z}$, using an identification $\left.W_{\overline{F_{v}}} \cong \mathbb{C}^{\times}\right)$.

3) A semisimple Galois representation $\rho: G_{F} \rightarrow \mathrm{GL}_{n}\left(\overline{\mathbb{Q}}_{\ell}\right)$ is said to be potentially abelian if there exists a finite extension $E / F$ such that $\left.\rho\right|_{G_{E}}$ is a direct sum of characters.

There is a strong link between algebraic Weil parameters and semisimple geometric potentially abelian $\ell$-adic representations as the following well-known proposition indicates. Recall that a continuous irreducible representation of a topological group is called primitive if it is not induced from any open finite index subgroup.

Proposition 7. 1) If $r: W_{F} \rightarrow \mathrm{GL}_{n}(\mathbb{C})$ is a primitive Weil parameter, then there exists a finite image representation $\sigma: G_{F} \rightarrow \mathrm{GL}_{n}(\mathbb{C})$ and a character $\chi: W_{F} \rightarrow \mathbb{C}^{\times}$such that $r=\sigma \otimes \chi$.

2) If $\rho: G_{F} \rightarrow \mathrm{GL}_{n}\left(\overline{\mathbb{Q}}_{\ell}\right)$ is primitive and potentially abelian, then there is a finite image representation $\tau: G_{F} \rightarrow \mathrm{GL}_{n}\left(\overline{\mathbb{Q}}_{\ell}\right)$ and a character $\epsilon: G_{F} \rightarrow \overline{\mathbb{Q}}_{\ell}^{\times}$ such that $\rho=\tau \otimes \epsilon$.

Proof. 1) We give the proof, following [Tat, §2.2.3]. As $W_{F}=\lim _{E} W_{E / F}$ and $\mathrm{GL}_{n}(\mathbb{C})$ has no small subgroups we can find a finite Galois extension $E / F$ such that $r$ factors through $W_{E / F}$. Then $W_{E}^{a b}$ is an abelian normal subgroup of $W_{E / F}$ so since $r$ is primitive Clifford's theorem tells us that $W_{E}^{a b}$ acts by scalars. Thus $\operatorname{proj} r$ factors through $\operatorname{Gal}(E / F)$. By Tate's result that $H^{2}\left(G_{F}, \mathbb{C}^{\times}\right)=1 \operatorname{proj} r$ lifts to representation $\sigma: G_{F} \rightarrow \mathrm{GL}_{n}(\mathbb{C})$ (necessarily of finite image and irreducible). As $r$ and $\sigma$ have the same projectivization, there is a character $\chi$ of $W_{F}$ such that $r=\sigma \otimes \chi$.

2) The proof is the same as 1 ), with a few minor changes. First, by assumption we can find $E / F$ finite such that $\left.\rho\right|_{G_{E}}$ is abelian. As in 1) Clifford's theorem implies that $\left.\rho\right|_{G_{E}}$ acts by scalars and hence that $\operatorname{proj} \rho$ factors through $\operatorname{Gal}(E / F)$. Using $\iota$ we get a projective representation to $\mathrm{PGL}_{n}(\mathbb{C})$ which we may lift as before to a 
finite image representation of $G_{F}$; using $\iota$ again we get a finite image irreducible representation $\tau: G_{F} \rightarrow \mathrm{GL}_{n}\left(\overline{\mathbb{Q}}_{\ell}\right)$ with $\operatorname{proj} \rho=\operatorname{proj} \tau$, and we may conclude that there is a character $\epsilon: G_{F} \rightarrow \overline{\mathbb{Q}}_{\ell}^{\times}$such that $\rho=\tau \otimes \epsilon$.

Remark 8. Of course the use of $\iota$ in the proof of 2 ) is unnecessary (and, as the author is well aware of, may seem offensive to some). A short argument with projective representations of finite groups shows that $\operatorname{proj} \rho$ takes values in $\operatorname{PGL}_{n}(\overline{\mathbb{Q}})$ and so rather than using $\iota$ it is enough to use the embeddings $\overline{\mathbb{Q}} \hookrightarrow \mathbb{C}$ and $\overline{\mathbb{Q}} \hookrightarrow \overline{\mathbb{Q}}_{\ell}$.

The next theorem we record is also well known; see e.g. [Far, $\S 1, \S 8]$ and the references within. The corollary is essentially (a weak version of) Far, Proposition 7.12]; we give a proof based on Proposition 7

Theorem 9. There exists a bijective correspondence between algebraic characters of $W_{F}$ and geometric $\ell$-adic characters of $G_{F}$ characterized by the property that if $\chi \leftrightarrow \epsilon$, then for $v \nmid \ell \infty,\left.\chi\right|_{W_{F v}}$ is unramified if and only if $\left.\epsilon\right|_{G_{F_{v}}}$ is unramified, and $\chi\left(\right.$ Frob $\left._{v}\right)=\iota\left(\epsilon\left(\right.\right.$ Frob $\left.\left._{v}\right)\right) \in \overline{\mathbb{Q}}$.

Moreover, if $\chi \leftrightarrow \epsilon$, there exists a (necessarily unique) integer $w$, the weight of $\chi($ or $\epsilon)$, such that:

1) For $v \nmid \ell \infty$ such that $\left.\chi\right|_{W_{F_{v}}}$ is unramified, all conjugates of $\chi\left(\right.$ Frob $\left._{v}\right)$ have Archimedean absolute value $q_{v}^{-w / 2}$, where $q_{v}$ is the size of the residue field of $F_{v}$.

2) For $v \mid \infty$, if $\left.\chi\right|_{W_{\overline{F_{v}}}}$ has the form $z \mapsto z^{p} \bar{z}^{q}$ (for some identification $W_{\overline{F_{v}}} \cong$ $\left.\mathbb{C}^{\times}\right)$, then $p+q=w$.

Corollary 10. 1) There exists a bijective correspondence $r \mapsto \rho_{r}, \rho \mapsto r_{\rho}$ between irreducible algebraic Weil parameters and irreducible potentially abelian geometric $\ell$-adic representations characterized by the property that if $r \leftrightarrow \rho$, then for $v \nmid$ $\left.\ell \infty r\right|_{W_{F_{v}}}$ is unramified if and only if $\left.\rho\right|_{G_{F_{v}}}$ is unramified, and $\operatorname{tr}\left(r\left(F_{r o b}\right)\right)=$ $\iota\left(\operatorname{tr}\left(\rho\left(F_{r o b}\right)\right)\right)$.

2) There exists a bijective correspondence between semisimple algebraic Weil parameters and semisimple potentially abelian geometric $\ell$-adic representations characterized by the property that if $r \leftrightarrow \rho$, then for $\left.v \nmid \ell \infty r\right|_{W_{F_{v}}}$ is unramified if and only if $\left.\rho\right|_{W_{F v}}$ is unramified, and $\operatorname{tr}\left(r\left(F_{r o b}\right)\right)=\iota\left(\operatorname{tr}\left(\rho\left(F_{r o b}\right)\right)\right)$.

Proof. 2) follows immediately from 1). To prove 1), note that it is clear for finite image representations on both sides; just use $\iota$. For characters this is Theorem 9. Thus for primitive representations we write $r=\sigma \otimes \chi, \rho=\tau \otimes \epsilon$ as in Proposition 7 and define $\rho_{r}=\rho_{\sigma} \otimes \rho_{\chi}$ and $r_{\rho}=r_{\tau} \otimes r_{\epsilon}$. Then for general irreducibles $r=\operatorname{Ind} r^{\prime}$, $\rho=$ Ind $\rho^{\prime}$ with $r^{\prime}, \rho^{\prime}$ primitive, set $\rho_{r}=$ Ind $\rho_{r^{\prime}}, r_{\rho}=\operatorname{Ind} r_{\rho^{\prime}}$; it is clear that these constructions do the job and are inverse to each other (note that $r=\operatorname{Ind} r^{\prime}$ is algebraic if and only if $r^{\prime}$ is algebraic).

3.2. Equidistribution laws. We begin this section with a well-known fact on the $L$-functions of Weil parameters, and hence of their associated Galois representations in the case when they are algebraic.

Proposition 11. Let $r: W_{F} \rightarrow \mathrm{GL}_{n}(\mathbb{C})$ be an irreducible unitary Weil parameter. Then the Artin $L$-function $L(s, r)$ has meromorphic continuation to all of $\mathbb{C}$. Moreover it is invertible (i.e. holomorphic and non-vanishing) on Res $\geq 1$ unless $r=|\cdot|{ }^{i \lambda}$ for some $\lambda \in \mathbb{R}$. 
Proof. When $n=1$ this is well known by class field theory. Let $n \geq 2$. If $r$ is primitive, then we write $r=\sigma \otimes \chi$ as in Proposition 7. Assume first that $\chi$ is $\epsilon|\cdot|^{i \lambda}$ for some finite order character $\epsilon$ and $\lambda \in \mathbb{R}$. Then without loss of generality $\epsilon=1$ (just incorporate it in $\sigma$ ) and $L(s, r)=L(s+i \lambda, \sigma)$ so it is enough to treat the case $r=\sigma$ finite image. By Brauer's theorem we may write $r$ as a virtual direct sum

$$
r=\bigoplus_{i \in I}\left(\operatorname{Ind}_{W_{E_{i}}}^{W_{F}} \epsilon_{i}\right)^{\oplus a_{i}}
$$

where the $E_{i} / F$ are finite, $\epsilon_{i}$ is a finite order character, $a_{i} \in \mathbb{Z}$ and $I$ is a finite indexing set. Thus $L(s, r)=\prod_{i} L\left(s, \epsilon_{i}\right)^{a_{i}}$ from which we deduce the meromorphic continuation and the invertibility, except possibly at $s=1$. The order of vanishing at $s=1$ is $-\sum_{i \in T} a_{i}$ where the sum runs over the set $T \subseteq I$ of $i$ such that $\epsilon_{i}=1$. By character theory of finite groups and Frobenius reciprocity

$$
0=\langle\operatorname{tr} r, 1\rangle=\sum_{i \in I} a_{i}\left\langle\epsilon_{i}, 1\right\rangle=\sum_{i \in T} a_{i}
$$

where $\langle-,-\rangle$ denotes the usual inner product of characters, hence $L(s, r)$ is invertible at $s=1$ as well.

Next if $\chi$ is not of the form $\epsilon|\cdot|{ }^{i \lambda}$, then it is not of this form when restricted to any open finite index subgroup. We have $r=\sigma \otimes \chi$ and use Brauer's theorem to write

$$
\sigma=\bigoplus_{i \in I}\left(\operatorname{Ind}_{W_{E_{i}}}^{W_{F}} \epsilon_{i}\right)^{\oplus a_{i}}
$$

with notation as before, hence

$$
r=\bigoplus_{i \in I}\left(\operatorname{Ind}_{W_{E_{i}}}^{W_{F}}\left(\left.\epsilon_{i} \otimes \chi\right|_{W_{E_{i}}}\right)\right)^{\oplus a_{i}}
$$

and $L(s, r)=\prod_{i} L\left(s,\left.\epsilon_{i} \otimes \chi\right|_{W_{E_{i}}}\right)^{a_{i}}$ and none of the $\left.\epsilon_{i} \otimes \chi\right|_{W_{E_{i}}}$ are of the form $|\cdot|^{i \lambda}$ which allows us to deduce the proposition in this case.

Finally when $r$ is not primitive, write $r=$ Ind $r^{\prime}$ with $r^{\prime}$ primitive and then $L(s, r)=L\left(s, r^{\prime}\right)$ (note that inducing $|\cdot|^{i \lambda}$ never produces an irreducible representation).

Next, define $W_{F}^{1}=K e r|\cdot|$ and $W_{F}^{a b, 1}=\operatorname{Ker}\left(|\cdot|: W_{F}^{a b} \rightarrow \mathbb{R}_{>0}\right)$.

Lemma 12. Let $r: W_{F} \rightarrow \mathrm{GL}_{n}(\mathbb{C})$ be a unitary Weil parameter. Let $E / F$ be a finite Galois extension such that $r$ factors through $W_{E / F}$. Then the image of $r$ is compact, hence closed in $\mathrm{GL}_{n}(\mathbb{C})$, and the following are equivalent:

1) $r\left(W_{E}^{a b, 1}\right)=r\left(W_{E}^{a b}\right)$.

2) $r\left(W_{F}^{1}\right)=r\left(W_{F}\right)$.

3) Write $\left.r\right|_{W_{E}}=\chi_{1} \oplus \ldots \oplus \chi_{n}$. Then for any $a_{1}, \ldots, a_{n} \in \mathbb{Z}, \chi_{1}^{a_{1}} \cdots \chi_{n}^{a_{n}} \neq\left.|\cdot|\right|^{i \lambda}$ for all $\lambda \in \mathbb{R}^{\times}$.

Proof. Recall that $W_{E}^{a b} \cong W_{E}^{a b, 1} \times \mathbb{R}_{>0}$ and that $W_{E}^{a b, 1}$ is compact. Thus $r\left(W_{E}^{a b, 1}\right)$ is compact and on $\mathbb{R}_{>0}, r$ is a unitary character and is hence trivial or maps surjectively onto $\mathrm{U}(1)$. It follows that $r\left(W_{E}^{a b}\right)$ is compact. Since $W_{E}^{a b}$ has finite index in $W_{E / F}$ it follows that the image of $r$ is compact. 
Let us now show that 1) and 2) are equivalent. To do this, we introduce $W_{E / F}^{1}$ which is the kernel of the norm map on $W_{E / F}$. It sits in an exact sequence

$$
1 \rightarrow W_{E}^{a b, 1} \rightarrow W_{E / F}^{1} \rightarrow \operatorname{Gal}(E / F) \rightarrow 1
$$

Note that $W_{E}^{a b, 1}=W_{E / F}^{1} \cap W_{E}^{a b}$ is normal in $W_{E / F}$, and also that 2) is equivalent to $r\left(W_{E / F}^{1}\right)=r\left(W_{E / F}\right)$. We have $W_{E / F} / W_{E / F}^{1} \cong W_{E}^{a b} / W_{E}^{a b, 1} \cong \mathbb{R}_{>0}$ and these surject onto $r\left(W_{E / F}\right) / r\left(W_{E / F}^{1}\right)$ and $r\left(W_{E}^{a b}\right) / r\left(W_{E}^{a b, 1}\right)$, so they are connected. Thus it suffices to show that if one is finite, then the other is as well. Assume 1). Then

$$
\operatorname{Gal}(E / F) \rightarrow \frac{r\left(W_{E / F}\right)}{r\left(W_{E}^{a b}\right)}=\frac{r\left(W_{E / F}\right)}{r\left(W_{E}^{a b, 1}\right)} \rightarrow \frac{r\left(W_{E / F}\right)}{r\left(W_{E / F}^{1}\right)}
$$

so 2) holds. Conversely, assume 2). Then

$$
\operatorname{Gal}(E / F) \rightarrow \frac{r\left(W_{E / F}^{1}\right)}{r\left(W_{E}^{a b, 1}\right)}=\frac{r\left(W_{E / F}\right)}{r\left(W_{E}^{a b, 1}\right)} \supseteq \frac{r\left(W_{E}^{a b}\right)}{r\left(W_{E}^{a b, 1}\right)}
$$

and hence 1) holds.

To finish the proof of the proposition it suffices to show that 1) and 3) are equivalent. First assume 1). $\left.r\right|_{W_{E}}$ takes values in $\mathrm{U}(1)^{n}$ and the integers $a_{1}, \ldots, a_{n}$ define character $\psi$ such that $\psi \circ r=\chi_{1}^{a_{1}} \cdots \chi_{n}^{a_{n}}$. Then $\psi\left(r\left(W_{E}^{a b}\right)\right)=\psi\left(r\left(W_{E}^{a b, 1}\right)\right)$ which implies $\psi \circ r \neq\left.|\cdot|\right|^{i \lambda}$ for all $\lambda \in \mathbb{R}^{\times}$as $|\cdot|^{i \lambda}\left(W_{E}^{a b}\right) \neq 1=|\cdot|^{i \lambda}\left(W_{E}^{a b, 1}\right)$ for $\lambda \in \mathbb{R}^{\times}$. Thus 1) implies 3). Conversely, assume that 3) holds and let $G=r\left(W_{E}^{a b}\right)$. As $W_{E}^{a b, 1}$ is compact, $r\left(W_{E}^{a b, 1}\right)$ is a closed subgroup of $G$. Assume for contradiction that $G / r\left(W_{E}^{a b, 1}\right) \neq 1$. As $\mathbb{R}_{>0} \rightarrow G / r\left(W_{E}^{a b, 1}\right)$ we must have $G / r\left(W_{E}^{a b, 1}\right)=\mathrm{U}(1)$ and hence we may find a non-trivial character $\psi$ on $G$ that is trivial on $r\left(W_{E}^{a b, 1}\right)$. Thus $\psi \circ r$ is non-trivial but trivial on $W_{E}^{a b, 1}$ and hence equal to $|\cdot|^{i \lambda}$ for some $\lambda \in \mathbb{R}^{\times}$. As $G$ is a closed subgroup of $\mathrm{U}(1)^{n}$ we may extend $\psi$ to a character on $\mathrm{U}(1)^{n}$ and hence there are integers $a_{1}, \ldots, a_{n}$ such that $\chi_{1}^{a_{1}} \cdots \chi_{n}^{a_{n}}=|\cdot|{ }^{i \lambda}$, a contradiction. This concludes the proof.

We may now prove an equidistribution result for Weil parameters.

Theorem 13. Let $r: W_{F} \rightarrow \mathrm{GL}_{n}(\mathbb{C})$ be a unitary Weil parameter and put $G=$ $r\left(W_{F}\right)$ (which is compact by Lemma 12). Assume that $G=r\left(W_{F}^{1}\right)$. Let $S$ be a finite set of places containing all infinite places such that $r$ is unramified outside $S$. Then the sequence $\left(r\left(F_{r o b}\right)\right)_{v \notin S}$ is equidistributed in the set of conjugacy classes of $G$ with respect to measure induced by the Haar probability measure on $G$.

Proof. As indicated, it suffices to show that for every irreducible non-trivial representation $\rho: G \rightarrow \mathrm{GL}_{m}(\mathbb{C})$, the Artin $L$-function $L(s, \rho \circ r)$ of the (necessarily irreducible) unitary Weil parameter $\rho \circ r$ has meromorphic continuation and is invertible on $R e s \geq 1$. By Proposition 11] it suffices to show that $\rho \circ r \neq|\cdot|{ }^{i \lambda}$ for any $\lambda \in \mathbb{R}$. As $r$ surjects onto $G \rho \circ r$ cannot be trivial, and since $(\rho \circ r)\left(W_{F}^{1}\right)=(\rho \circ r)\left(W_{F}\right)$, $\rho \circ r \neq|\cdot|^{i \lambda}$ for all $\lambda \in \mathbb{R}^{\times}$. 
3.3. Applications to Galois representations and abelian varieties. In this section we apply Theorem 13 to get equidistribution laws for abelian varieties that become isogenous over some field extension to a product of abelian varieties with CM. First we give results purely in terms of Galois representations.

Lemma 14. Let $\rho: G_{F} \rightarrow \mathrm{GL}_{n}\left(\overline{\mathbb{Q}}_{\ell}\right)$ be a semisimple potentially abelian geometric $\ell$-adic representation that is pure of weight $w \in \mathbb{Z}$, and let $r$ be the associated Weil parameter. Then:

1) $r \otimes|\cdot|^{-w / 2}$ is unitary.

2) $\left(r \otimes|\cdot|^{-w / 2}\right)\left(W_{F}\right)=\left(r \otimes|\cdot|^{-w / 2}\right)\left(W_{F}^{1}\right)$.

Proof. 1) First note that if $E / F$ is any finite extension, then $\rho$ is pure of weight $w$ if and only if $\left.\rho\right|_{G_{E}}$ is pure of weight $w$, and $r \otimes|\cdot|^{-w / 2}$ is unitary if and only if $\left.r\right|_{W_{E}} \otimes|\cdot|^{-w / 2}$ is unitary (as being unitary is equivalent to compactness of the image, by Lemma 12 and the unitary trick). Thus we are reduced to the case when $\rho$ is a sum of characters, which directly reduces to the case of a single character. Then as is well known there exists a unique $\mu \in \mathbb{R}$ such that $r \otimes|\cdot|^{-\mu / 2}$ is unitary. For $v \nmid \ell \infty$ such that $r$ is unramified, we must then have $\left|\iota\left(\rho\left(F_{r o b}\right)\right)\right|=\left|r\left(F_{r o b}\right)\right|=q_{v}^{-\mu / 2}$ (where the absolute value denotes the complex absolute value) and hence $\mu=w$ as desired.

2) By Lemma 12 we are again reduced to the case when $\rho=\epsilon_{1} \oplus \cdots \oplus \epsilon_{n}$ is a sum of characters and if we write $r=\chi_{1} \oplus \cdots \oplus \chi_{n}$, we want to show that for any $a_{1}, \ldots, a_{n} \in \mathbb{Z},\left(\chi_{1}|\cdot|^{-w / 2}\right)^{a_{1}} \cdots\left(\chi_{n}|\cdot|^{-w / 2}\right)^{a_{n}} \neq|\cdot|^{i \lambda}$ for all $\lambda \in \mathbb{R}^{\times}$, i.e., that $\chi_{1}^{a_{1}} \cdots \chi_{n}^{a_{n}} \neq|\cdot|^{\left(\sum a_{i}\right)(w / 2)+i \lambda}$. Since each $\chi_{i}$ is algebraic, we see that the left-hand side is algebraic, but the right-hand side is only algebraic if $\lambda=0$.

Corollary 15. Let $\rho: G_{F} \rightarrow \mathrm{GL}_{n}\left(\overline{\mathbb{Q}}_{\ell}\right)$ be a semisimple potentially abelian geometric $\ell$-adic representation that is pure of weight $w \in \mathbb{Z}$, and let $S$ be a finite set of places containing all infinite places and places above $\ell$ such that $\rho$ is unramified outside $S$. Let $r$ be the Weil parameter associated with $\rho$. Then the elements $r\left(F_{r o b}\right) q_{v}^{w / 2}$ all lie in some compact group $G \subseteq \mathrm{GL}_{n}(\mathbb{C})$ and are equidistributed in the space of conjugacy classes of $G$ (with respect to normalized Haar measure on $G$ ).

Proof. By the lemma we may apply Theorem 13 to $r \otimes|\cdot|^{-w / 2}$.

Applying this to the Tate module of an abelian variety that becomes isogenous to a product of abelian varieties with $\mathrm{CM}$ over a finite extension of $F$, we get an equidistribution result. From the definition of $G$ and the $r\left(F r o b_{v}\right) q^{w / 2}$ it is not clear that this data agrees with $S T_{A}$ and the conjugacy classes defined in section 2.1. Next, we will prove the Sato-Tate conjecture in the form of [FKRS] for products (up to isogeny) of CM abelian varieties.

Proposition 16. Let $A / F$ be an abelian variety that becomes isogenous to a product of abelian varieties with CM over a finite extension of $F$. Then the Sato-Tate conjecture holds for A. In particular, the Sato-Tate conjecture holds for abelian surfaces of type $\boldsymbol{D}$ or $\boldsymbol{F}$.

Proof. We follow the general strategy outlined in section 2.2 , Let $R$ be an irreducible representation of $S T_{A}$. By the remarks in the last paragraphs of section 4.1 we may extend $R$ to an algebraic representation of $G_{\ell}^{Z a r}$, which we also denote by $R$, and $R \circ \rho_{A, \ell}$ is a pure, semisimple, potentially abelian geometric Galois representation. By Corollary 10. Theorem 13 and Lemma 14 we may now conclude. 
Remark 17. Let $A / F$ be an abelian variety satisfying the hypotheses of Proposition 16. with $\ell$-adic Tate module $\rho=\rho_{A, \ell}$ and corresponding Weil parameter $r$. Associated with $A$ we have groups $G$ and $S T_{A}$ together with conjugacy classes $\left(r\left(F r o b_{v}\right) q^{1 / 2}\right)_{v}$ and $(s(v))_{v}$, and we have proved equidistribution results for both. When viewed as elements of $\mathrm{GL}_{2 g}(\mathbb{C}), r\left(F_{r o b}\right) q^{1 / 2}$ and $s(v)$ have the same characteristic polynomial. It is not clear (at least to the author) that $G=S T_{A}$ and $r\left(F_{r o b}\right) q^{1 / 2}=s(v)$, though it seems a natural guess. Unfortunately we have not been able to prove this equality. The issue (at least for the author of this paper) seems to be the inexplicit nature of the construction of the global Weil group for number fields.

\section{Abelian surfaces potentially of $\mathrm{GL}_{2}$-Type OVER TOTALLY REAL FIELDS}

In this section we will deal with a number of cases where the abelian surface $A / F$ has a two-dimensional commutative semisimple $\mathbb{Q}$-algebra $K \subseteq \operatorname{End}_{\overline{\mathbb{Q}}}(A)^{0}$. For technical reasons we need to assume that $K \subseteq \operatorname{End}_{F^{\prime}}(A)^{0}$ for a certain totally real field $F^{\prime} \supseteq F$ (forcing $F$ to be totally real as well). This is analogous to the restriction to elliptic curves over totally real fields in our current knowledge of the Sato-Tate conjecture for non-CM elliptic curves. We will start off by recalling some generalities from $\mathrm{Rib}$ on the Tate modules of abelian varieties of $\mathrm{GL}_{2}$-type, and then we will prove the Sato-Tate conjecture case by case for cases $\mathbf{B}, \mathbf{C}$ and $\mathbf{E}$ under the above-mentioned hypotheses.

4.1. Some generalities. To simplify terminology, let us make the following nonstandard definition (cf. [Rib, $\S 2]$ in the case $F=\mathbb{Q}$ for the standard definition).

Definition 18. Let $A / F$ be an abelian variety. We say that $A$ is of $\mathrm{GL}_{2}$-type if it is isogenous over $F$ to a product $A_{1} \times \cdots \times A_{r}$ of simple abelian varieties $A_{i}$ over $F$ and there is a number field $K_{i} \subseteq \operatorname{End}_{F}\left(A_{i}\right)^{0}$ of degree $\operatorname{dim} A_{i}$ for each $i=1, \ldots, r$.

We will write $K$ for the product $\prod_{i=1}^{r} K_{i}$ viewed as a subalgebra of $\operatorname{End}_{F}(A)^{0}$. If $A / F$ is an abelian variety, note that if there is a commutative semisimple subalgebra $K \subseteq \operatorname{End}_{F}(A)^{0}$ of $\operatorname{dimension} \operatorname{dim} A$, then $A / F$ is of $\mathrm{GL}_{2}$-type if each factor of $A$ corresponding to a simple factor of $K$ is simple over $F$.

Remark 19. With the usual definition of $\mathrm{GL}_{2}$-type (corresponding to requiring $r=1$ in the above definition) one would say that an abelian variety $A$ satisfying the above definition is isogenous to a product of abelian varieties of $\mathrm{GL}_{2}$-type. We have chosen to relax the standard definition to avoid the cumbersome terminology "isogenous to a product of abelian varieties of $\mathrm{GL}_{2}$-type"; we apologize for this but hope that it will not cause the reader too much confusion.

Let $A$ be an abelian surface of $\mathrm{GL}_{2}$-type over a totally real field $F$ with $K \subseteq$ $\operatorname{End}_{F}(A)^{0}$ of a two-dimensional commutative semisimple algebra. We have two cases corresponding to whether $A$ is simple and $K$ is a field, or $K=\mathbb{Q} \times \mathbb{Q}$ and $A$ is isogenous to a product of elliptic curves. If $A$ is simple, the following results are recorded in $[\mathrm{Rib}, \S 3]$ when $F=\mathbb{Q}$, but they extend with the same proofs to general totally real $F$. We refer to [BLGGT, $\S 5]$ for the definition of a weakly compatible system. 
Proposition 20. Let $A$ be a simple abelian surface of $\mathrm{GL}_{2}$-type over a totally real field $F$ and let $K \subseteq \operatorname{End}_{F}(A)^{0}$ be a quadratic field. Let $\ell$ be a prime number. The $\ell$-adic (rational) Tate module $\rho_{A, \ell}: G_{F} \rightarrow \mathrm{GL}_{4}\left(\overline{\mathbb{Q}}_{\ell}\right)$ lands inside $\mathrm{GL}_{2}\left(K \otimes_{\mathbb{Q}} \overline{\mathbb{Q}}_{\ell}\right)$ and we may decompose it into two two-dimensional pieces using the two embeddings $K \hookrightarrow \overline{\mathbb{Q}}_{\ell}$. The two-dimensional pieces fit together into a weakly compatible system $\left(\rho_{A, \lambda}: G_{F} \rightarrow \mathrm{GL}_{2}\left(\bar{K}_{\lambda}\right)\right)_{\lambda}$ where $\lambda$ ranges over the finite places of $K$ (or just embeddings $K \hookrightarrow \overline{\mathbb{Q}}_{\ell}$ for all primes $\left.\ell\right)$.

In the following we use the association $\ell \leftrightarrow \lambda$ without further comment. The compatible system $\left(\rho_{A, \lambda}\right)_{\lambda}$ satisfies the following properties (see [Rib, Lemmas 3.1 and 3.2] for properties 1) and 2)):

1) Assume that $K$ is a real quadratic field. Then $\operatorname{det} \rho_{A, \lambda}=\chi_{\ell}$, the $\ell$-adic cyclotomic character.

2) Assume that $K$ is an imaginary quadratic field. Then $\operatorname{det} \rho_{A, \lambda}=\epsilon \chi_{\ell}$, where $\epsilon: G_{F} \rightarrow K^{\times}$is a finite order character (independent of $\lambda$ ).

3) In either case $\rho_{A, \lambda}$ is totally odd, regular with Hodge-Tate weights 0 and -1 (independent of embeddings and choice of places), and the compatible system is pure of weight -1 .

When $A$ is isogenous over $F$ to a product of elliptic curves the obvious analogue of parts 1) and 3) of the above proposition holds (one might view the algebra $\mathbb{Q} \times \mathbb{Q}$ as "totally real"). We also record a conjugate self-duality result in the case when $K$ is imaginary quadratic.

Proposition 21 ([ $\mathrm{Rib}$, Proposition 3.4]). We use the notation of Proposition 20 and assume in addition that $K$ is imaginary quadratic. Then $\rho_{A, \lambda_{1}} \cong \rho_{A, \lambda_{2}} \otimes \epsilon$, where $\epsilon$ denotes the character in Proposition 20 2) and the $\lambda_{i}$ are the two distinct embeddings $K \hookrightarrow \overline{\mathbb{Q}}_{\ell}$.

Finally, let us recall the following from the discussion leading up to the definition of the Sato-Tate group: We have an exact sequence

$$
1 \rightarrow G_{\ell}^{1, Z a r} \rightarrow G_{\ell}^{Z a r} \rightarrow \mathbb{G}_{m} \rightarrow 1
$$

and $G_{\ell}^{Z a r}$ contains the central $\mathbb{G}_{m}$ inside $\mathrm{GL}_{4}$. Since the similitude character becomes $z \mapsto z^{2}$ when restricted to the central $\mathbb{G}_{m}$ we have an isogeny $G_{\ell}^{1, Z a r} \times$ $\mathbb{G}_{m} \rightarrow G_{\ell}^{Z a r}$. This gives us a surjection $G^{1} \times \mathbb{C}^{\times} \rightarrow G$ on $\mathbb{C}$-points with kernel $\{1,(-1,-1)\}$. Since $S T_{A}$ is a compact real form of the complex reductive group $G^{1}$ we see that any (complex, finite-dimensional) irreducible representation of $S T_{A}$ may be extended to an irreducible algebraic representation of $G$, hence of $G_{\ell}^{Z a r}\left(\overline{\mathbb{Q}}_{\ell}\right)$, by choosing a compatible character of the central $\mathbb{G}_{m}$.

This has the following important consequence: Let $r$ be an irreducible representation of $S T_{A}$ and let $w \in \mathbb{Z}$ be such that $r(-1)=(-1)^{w}$. Then $z \mapsto z^{w}$ is compatible with $r$ and the data $(r, w)$ defines an irreducible algebraic representation $R(w)$ of $G_{\ell}^{Z a r}\left(\overline{\mathbb{Q}}_{\ell}\right)$. Thus we get a weakly compatible system of Galois representations $R(w) \circ \rho_{A, \ell}$ that are essentially self-dual, totally odd and irreducible (importantly) and satisfies

$$
L^{S}(s, r, A)=L^{S}\left(s+w / 2, R(w) \circ \rho_{A, \ell}\right)
$$

(directly from the definition of the two Euler products). 
4.2. Galois type $\mathbf{B}\left[C_{1}\right]$. Recall that we are using the presentation $\operatorname{USp}(4)=$ $\left\{A \in \mathrm{U}(4) \mid A^{t} S^{\prime} A=S^{\prime}\right\}$. Case $\mathbf{B}$ corresponds to $S T_{A}^{0}=\mathrm{SU}(2) \times \mathrm{SU}(2)$, which we may embed into $\mathrm{USp}(4)$ by

$$
(A, B) \mapsto\left(\begin{array}{cc}
A & 0 \\
0 & B
\end{array}\right) .
$$

The reference for the group theory is FKRS, §3.6].

In the case of Galois type $\mathbf{B}\left[C_{1}\right]$ the Sato-Tate group is simply $\mathrm{SU}(2) \times \mathrm{SU}(2)$ and its irreducible representations are $r_{k, l}=S y m^{k}(S t) \otimes S y m^{l}(S t)$ for non-negative integers $k, l$, where $S t$ denotes the standard two-dimensional representation of any subgroup of $\mathrm{GL}_{2}$. Let $A / F$ be an abelian surface of Galois type $\mathbf{B}\left[C_{1}\right]$. There are two cases to consider; either $A$ is isogenous to the product of elliptic curves over $F$ without $\mathrm{CM}$ that do not become isogenous over any finite extension of $F$, or $A$ has real multiplication defined over $F$ by a real quadratic field $K$. In the first case, the Sato-Tate conjecture is known and due to Harris ([Har, Theorem 5.4]), modulo certain hypotheses that have subsequently been verified (see [BGHT] for a discussion and references). It remains to deal with the second case. The proof is the same as that of Harris in the first case (up to a few minor details and the general remark that we use more powerful potential automorphy theorems).

Proposition 22. Let $A$ be an abelian surface over $F$ such that $\operatorname{End}_{F}(A)^{0}=$ $\operatorname{End}_{\overline{\mathbb{Q}}}(A)^{0}=K$ is a two-dimensional semisimple algebra over $\mathbb{Q}$. Then the SatoTate conjecture holds for $A$.

Proof. As mentioned above, $K$ is either $\mathbb{Q} \times \mathbb{Q}$ or a real quadratic field, the first case being treated by Harris, so we may assume that $K$ is real quadratic (the proof below also works with the obvious modifications). Consider the regular, totally odd and essentially self-dual weakly compatible system $\left(\rho_{A, \lambda}\right)_{\lambda}$ given by Proposition 20. For any $m \geq 0$, the weakly compatible system $\left(\operatorname{Sym}^{m} \rho_{A, \lambda}\right)_{\lambda}$ inherits these properties and moreover is irreducible (since the $\rho_{A, \lambda}\left(G_{F}\right)$ is open in its Zariski closure, which is $\mathrm{GL}_{2}\left(\overline{\mathbb{Q}}_{\ell}\right)$ ) so we may apply [BLGGT, Theorem 5.4.1] to deduce that there is a Galois, totally real extension $F^{\prime} / F$ such that $\left(S y m^{m} \rho_{A, \lambda}\right)_{\lambda}$ becomes cuspidal automorphic after restriction to $F^{\prime}$.

Next, let us consider the partial $L$-function

$$
L^{S}\left(s, r_{k, l}, A\right)=\prod_{v \notin S} \frac{1}{\operatorname{det}\left(1-r_{k, l}(s(v)) q_{v}^{-s}\right)}
$$

which we want to show is invertible on $\operatorname{Re} s \geq 1$ when $(k, l) \neq(0,0)$. We may extend $r_{k, l}$ to a representation $R_{k, l}$ of $G_{\ell}^{Z a r}$ as described in the previous section. Here let us be explicit and, since $G_{\ell}^{Z a r} \subseteq \mathrm{GL}_{2}\left(\overline{\mathbb{Q}}_{\ell}\right) \times \mathrm{GL}_{2}\left(\overline{\mathbb{Q}}_{\ell}\right)$, define $R_{k, l}$ by restricting $S y m^{k} \otimes S y m^{l}$. Then we have

$$
\begin{aligned}
L^{S}\left(s, R_{k, l}, A\right) & =L^{S}\left(s+(k+l) / 2, R_{k, l} \circ r_{A, \ell}\right) \\
& =L^{S}\left(s+(k+l) / 2, \operatorname{Sym}^{k} \rho_{A, \lambda_{1}} \otimes \operatorname{Sym}^{l} \rho_{A, \lambda_{2}}\right)
\end{aligned}
$$

where we let $\lambda_{1}$ and $\lambda_{2}$ denote the two embeddings $K \hookrightarrow \overline{\mathbb{Q}}_{\ell}$. By Brauer's theorem, as in e.g. the proof of [HSBT, Theorem 4.2], the invertibility of this $L$-function follows from that of

$$
L^{S}\left(s+(k+l) / 2, \operatorname{Sym}^{k}\left(\left.\rho_{A, \lambda_{1}}\right|_{G_{E}}\right) \otimes \operatorname{Sym}^{l}\left(\left.\rho_{A, \lambda_{2}}\right|_{G_{E}}\right)\right)
$$


for arbitrary subextensions $F^{\prime} / E / F$ such that $F^{\prime} / E$ is solvable, and cuspidal automorphy of $\left(S y m^{m} \rho_{A, \lambda}\right)_{\lambda}$ over $F^{\prime}$ implies cuspidal automorphy over $E$ by the theory of cyclic base change ( $\mathrm{AC}]$ ). Thus, Rankin-Selberg theory (as in the proof of $\mathrm{Har}$, Theorem 5.3]) implies that $L^{S}\left(s+(k+l) / 2, \operatorname{Sym}^{k}\left(\left.\rho_{A, \lambda_{1}}\right|_{G_{E}}\right) \otimes \operatorname{Sym}^{l}\left(\left.\rho_{A, \lambda_{2}}\right|_{G_{E}}\right)\right)$ is invertible, as long as $\operatorname{Sym}^{k}\left(\left.\rho_{A, \lambda_{1}}\right|_{G_{E}}\right) \not \operatorname{Sym}^{l}\left(\left.\rho_{A, \lambda_{2}}\right|_{G_{E}}\right)$ (use essential selfduality and the fact that the multiplier of $\rho_{A, \lambda}$ is the cyclotomic character $\left.\chi_{\ell}\right)$. Then, arguing as in the paragraph before [Har, Theorem 5.3] (using our knowledge of $\operatorname{End}_{\overline{\mathbb{Q}}}(A)$ and the open image theorem for the $\left.\rho_{A, \lambda}\right)$, we deduce that if $\operatorname{Sym}^{k}\left(\left.\rho_{A, \lambda_{1}}\right|_{G_{E}}\right) \cong \operatorname{Sym}^{l}\left(\left.\rho_{A, \lambda_{2}}\right|_{G_{E}}\right)$, then there exists a finite extension $E^{\prime} / F$ such that $\left.\left.\rho_{A, \lambda_{1}}\right|_{G_{E^{\prime}}} \cong \rho_{A, \lambda_{2}}\right|_{G_{E^{\prime}}}$, but this contradicts the fact that $\operatorname{End}_{\overline{\mathbb{Q}}}(A)^{0}=K$ by Faltings's theorem.

4.3. Galois type $\mathbf{B}\left[C_{2}\right]$. Here $S T_{A}=N(\mathrm{SU}(2) \times \mathrm{SU}(2))=\langle\mathrm{SU}(2) \times \mathrm{SU}(2), J\rangle \subseteq$ $\mathrm{USp}(4)$. Note that $S T_{A}$ has two components as $J^{2}=I d_{4}$. Let us start by recording the following standard consequence of Clifford's theorem which will be used numerous times throughout this paper.

Lemma 23. Let $G$ and $H$ be topological groups with $H \subseteq G$ a subgroup of index 2 and let $x \in G \backslash H$ be any element. If $r$ is a continuous finite-dimensional irreducible representation of $H$ we define its twist $r^{x}$ by $r^{x}(h)=r\left(x h x^{-1}\right)$. Then $r$ extends to a representation of $G$ if and only if $r \cong r^{x}$, in which case it extends to exactly two non-isomorphic representations (one is obtained from the other by twisting by the non-trivial character of $G / H)$ and all the other irreducible representations of $G$ are of the form $\operatorname{Ind}_{H}^{G} r$ where $r$ is an irreducible representation of $H$ such that $r \neq r^{x}$.

We will use it here with $H=S T_{A}^{0}, G=S T_{A}$. Given $(A, B) \in S T_{A}^{0}$, we have that $J(A, B) J^{-1}=\left(J_{2} B J_{2}^{-1}, J_{2} A J_{2}^{-1}\right)$. Let $r_{k, l}=S y m^{k}(S t) \otimes S y m^{l}(S t)$ be an irreducible representation of $S T_{A}^{0}$. Then since $J_{2} \in \mathrm{SU}(2)$ we see that $r_{k, l}^{J} \cong r_{l, k}$. Hence $r_{k, l}$ extends if and only if $k=l$ and the lemma gives us all the irreducible representations of $S T_{A}$. For $k \neq l$, we put $s_{k, l}=I n d_{H}^{G} r_{k, l}$, for $k=l$ we have two representations $s_{k}^{1}, s_{k}^{2}$ that extend $r_{k, k}$. We make the convention that $s_{0}^{1}$ is the trivial representation.

Now let us consider an abelian surface $A / F$ of type $\mathbf{B}\left[C_{2}\right]$. This means that $L / F$ has degree two, $\operatorname{End}_{F}(A)^{0}=\mathbb{Q}$ and $\operatorname{End}_{L}(A)^{0}=\operatorname{End}_{\overline{\mathbb{Q}}}(A)^{0}$ is a two-dimensional semisimple algebra over $\mathbb{Q}$. Thus $\rho_{A, \ell}$ is irreducible but $\left.\rho_{A, \ell}\right|_{G_{L}}$ is a sum of two two-dimensional irreducible representations that do not become either isomorphic or reducible upon further extension of $F$.

Proposition 24. Let $A / F$ be an abelian surface of Galois type $\boldsymbol{B}\left[C_{2}\right]$ and assume that $L$ is totally real. Then the Sato-Tate conjecture holds for A.

Proof. We have an exact sequence

$$
1 \rightarrow G_{\ell}^{Z a r}\left(A_{L}\right)\left(\overline{\mathbb{Q}}_{\ell}\right) \rightarrow G_{\ell}^{Z a r}(A)\left(\overline{\mathbb{Q}}_{\ell}\right) \rightarrow \mathbb{Z} / 2 \mathbb{Z} \rightarrow 1 .
$$

Consider first the representation $s_{k, l}$ for $k \neq l$. Note that $s_{k, l}(-1)=r_{k, l}(-1)=$ $(-1)^{k+l}$, this gives us an algebraic representation of $G_{\ell}^{Z a r}(A)\left(\overline{\mathbb{Q}}_{\ell}\right)$, which we will denote by $S_{k, l}$, satisfying $S_{k, l}=\operatorname{Ind}_{G_{\ell}^{Z a r}\left(A_{L}\right)\left(\overline{\mathbb{Q}}_{\ell}\right)}^{G_{\text {Zar }}^{Z}(A)\left(\overline{\mathbb{Q}}_{\ell}\right)} R_{k, l}$ where the $R_{k, l}$ on the righthand side is the representation as in Proposition 22, Thus

$$
L^{S}\left(s, s_{k, l}, A\right)=L^{S}\left(s+(k+l) / 2, S_{k, l} \circ \rho_{A, \ell}\right)=L^{S}\left(s, r_{k, l}, A_{L}\right)
$$

and hence it is invertible by the proof of Proposition 22 . 
Next we let $k \geq 1$ and consider $s_{k}^{i}$. We have $s_{k}^{i}(-1)=s_{k, k}(-1)=(-1)^{2 k}$ so we may choose $w=2 k$ and extend $R_{k}^{i}$ to an algebraic representation $S_{k}^{i}$ of $G_{\ell}^{Z a r}(A)\left(\overline{\mathbb{Q}}_{\ell}\right)$ with $L^{S}\left(s, s_{k}^{i}, A\right)=L^{S}\left(s+k, S_{k}^{i} \circ \rho_{A, \ell}\right)$. The invertibility of this $L$ function follows from that of $L^{S}\left(s+k,\left.S_{k}^{i} \circ \rho_{A, \ell}\right|_{G_{E}}\right)=L^{S}\left(s+k,\left.r_{k, k} \circ \rho_{A, \ell}\right|_{G_{E}}\right)$ by Brauer's theorem, where $E$ is a subextension of a totally real Galois extension $F^{\prime} / L$, and moreover $F^{\prime} / F$ is Galois as well. We now apply the proof of Proposition 22 to conclude, noting that the extra condition that $F^{\prime} / F$ is Galois as well is allowed in BLGGT, Theorem 5.4.1].

Finally we need to consider $L^{S}\left(s, s_{0}^{2}, A\right)$. Since the composition $G_{F} \rightarrow$ $G_{\ell}^{Z a r}(A)\left(\overline{\mathbb{Q}}_{\ell}\right) \rightarrow \mathbb{Z} / 2 \mathbb{Z}$ is surjective with kernel $G_{L} L^{S}\left(s, s_{0}^{2}, A\right)$ is the Hecke $L$ function associated with the non-trivial character of $\operatorname{Gal}(L / F)$, hence invertible.

4.4. Type C. In this case only the Galois type $\mathbf{C}\left[C_{2}\right]$ is possible over a totally real field. We are still using the presentation $\mathrm{USp}(4)=\left\{A \in \mathrm{U}(4) \mid A^{t} S^{\prime} A=S^{\prime}\right\}$. We let $\mathrm{U}(1) \times \mathrm{SU}(2)$ be embedded into $\mathrm{USp}(4)$ by

$$
(u, A) \mapsto\left(\begin{array}{ccc}
u & & \\
& \bar{u} & \\
& & A
\end{array}\right) .
$$

$S T_{A}$ may then be described as $\langle\mathrm{U}(1) \times \mathrm{SU}(2), a\rangle$. Thus $S T_{A}=N(\mathrm{U}(1)) \times \mathrm{SU}(2)$ where $N(\mathrm{U}(1))$ denotes the normalizer of $\mathrm{U}(1)$ in $\mathrm{SU}(2)$, embedded via

$$
u \mapsto\left(\begin{array}{cc}
u & \\
& \bar{u}
\end{array}\right)
$$

The irreducible non-trivial representations of $N(\mathrm{U}(1))$ are of the form $r_{k}=$ $\operatorname{Ind} d_{\mathrm{U}(1)}^{N(\mathrm{U}(1))}\left(u \mapsto u^{k}\right)$ for $k \in \mathbb{Z}$ non-zero (use e.g. Lemma 23). If we let $r_{0}^{0}$ denote the trivial representation of $N(\mathrm{U}(1))$ and $r_{0}^{1}$ denotes the lift of the non-trivial character of $N(\mathrm{U}(1)) / \mathrm{U}(1)$, we deduce that the irreducible representations of $S T_{A}$ are of the form $r_{k} \otimes S y m^{l} S t$ for $k \in \mathbb{Z} \backslash\{0\}, l \in \mathbb{Z}_{\geq 0}$ and $r_{0}^{\epsilon} \otimes S y m^{l} S t$ for $\epsilon \in\{0,1\}$.

Consider an abelian variety $A / F$ of type $\mathbf{C}\left[C_{2}\right] . A$ is then isogenous to a product $E_{1} \times E_{2}$ of elliptic curves over $F$ ([FKRS, Proposition 4.5]) where we may take $E_{1}$ to have CM (defined over $L$ but not over $F$ ) and $E_{2}$ to have no CM.

Proposition 25. Let $A / F$ be an abelian surface of Galois type $C\left[C_{2}\right]$ and assume that $F$ is totally real (and hence $L$ is a CM field). Then the Sato-Tate conjecture holds for $A$.

Proof. We have $\rho_{A, \ell}=\rho_{E_{1}, \ell} \oplus \rho_{E_{2}, \ell}$ and $\rho_{E_{1}, \ell}=I n d_{G_{L}}^{G_{F}} \psi_{\ell}$ for some weakly compatible system of weight one essentially conjugate-self-dual algebraic Hecke characters $\psi_{\ell}$. From this one sees that for $k \geq 1, l \geq 1$,

$$
L^{S}\left(s, r_{k} \otimes \operatorname{Sym}^{l} S t, A\right)=L^{S}\left(s+(k+l) / 2, \operatorname{Sym}^{l}\left(\left.\rho_{E_{2}, \ell}\right|_{G_{L}}\right) \otimes \psi_{\ell}^{k}\right)
$$

and the $\left(\operatorname{Sym}^{l}\left(\left.\rho_{E_{2}, \ell}\right|_{G_{L}}\right) \otimes \psi_{\ell}^{k}\right)_{\ell}$ form a weakly compatible system of irreducible essentially conjugate-self-dual regular Galois representations which we may apply [BLGGT, Theorem 5.4.1] to deduce that it is potentially automorphic; invertibility 
of $L^{S}\left(s, r_{k} \otimes S y m^{l} S t, A\right)$ follows as usual from Brauer's theorem. For $k \geq 1, l=0$ we have

$$
L^{S}\left(s, r_{k} \otimes 1, A\right)=L^{S}\left(s+l / 2, \psi_{\ell}^{k}\right)
$$

and the result follows. For $l \geq 1, L^{S}\left(s, r_{0}^{\epsilon} \otimes \operatorname{Sym}^{l} S t, A\right)=L^{S}\left(s, S y m^{l} r_{E_{2}, \ell} \otimes \chi\right)$ where $\chi$ is a character of $\operatorname{Gal}(L / F)$ (trivial if and only if $\epsilon=0$ ); invertibility follows from potential automorphy of $S y m^{l} r_{E_{2}, \ell}$ and Brauer's theorem. Finally, $L^{S}\left(s, r_{0}^{1} \otimes 1, A\right)$ is the $L$-function of the non-trivial character of $\operatorname{Gal}(L / F)$, hence invertible.

4.5. Galois types $\mathbf{E}\left[C_{n}\right], n=1,3,4,6, \mathbf{E}\left[C_{2}, \mathbb{R} \times \mathbb{R}\right]$ and $\mathbf{E}\left[C_{2}, \mathbb{C}\right]$. We now switch presentation of $\mathrm{USp}(4)$ and instead use $\mathrm{USp}(4)=\left\{A \in \mathrm{U}(4) \mid A^{t} S A=S\right\}$. For type $E$ one has $S T_{A}^{0}=\mathrm{SU}(2)$. We embed $\mathrm{U}(2)$ (and hence $\mathrm{SU}(2)$ ) into $\mathrm{USp}(4)$ via

$$
A \mapsto\left(\begin{array}{cc}
A & \\
& \bar{A}
\end{array}\right) .
$$

The Galois types $\mathbf{E}\left[C_{n}\right], n=1,3,4,6, \mathbf{E}\left[C_{2}, \mathbb{R} \times \mathbb{R}\right]$ and $\mathbf{E}\left[C_{2}, \mathbb{C}\right]$ correspond to the Sato-Tate groups $E_{n}, n=1,3,4,6, J\left(E_{1}\right)$ and $E_{2}$ respectively, where the groups $E_{n}$ are defined as $\left\langle\mathrm{SU}(2), e^{\pi i / n}\right\rangle$ and $J\left(E_{1}\right)=\langle\mathrm{SU}(2), J\rangle$. Here $e^{\pi i / n}$ denotes the element $\operatorname{diag}\left(e^{\pi i / n}, e^{\pi i / n}, e^{-\pi i / n}, e^{-\pi i / n}\right) \in \mathrm{USp}(4)$. As $J$ commutes with $\mathrm{SU}(2)$ $J\left(E_{1}\right)$ is isomorphic to $\mathrm{SU}(2) \times C_{2}$.

For the groups $E_{n}$ the irreducible representations are of the form $r_{k, w}=S y m^{k} S t \otimes$ $\chi_{w}$, where $k \in \mathbb{Z}_{\geq 0}, w \in \mathbb{Z} / 2 n \mathbb{Z}, k \equiv w(2)$ and $\chi_{w}$ is the character $z \mapsto z^{w}$ of $\mu_{2 n}$. $E_{n}$ is then the group

$$
\mathrm{U}_{n}(2)=\left\{A \in \mathrm{U}(2) \mid \operatorname{det} A \in \mu_{n}\right\}
$$

embedded in $\mathrm{USp}(4)$ via the above embedding of $\mathrm{U}(2)$. For $J\left(E_{1}\right)$ the irreducible representations are of the form $r_{k, w}=S y m^{k} S t \otimes \chi_{w}, k$ integer $\geq 0$ and $w \in 0,1$, with $w=0$ corresponding to the trivial character of $C_{2}$.

Arithmetically these cases correspond to abelian surfaces $A$ such that $\operatorname{End}_{L}(A)^{0}$ is a quaternion algebra over $\mathbb{Q}$ and, when $F \neq L, \operatorname{End}_{F}(A)^{0}$ is a quadratic field or $\mathbb{Q} \times \mathbb{Q}$. This follows from [FKRS, Proposition 4.7], after we have made some remarks regarding the formulation of that proposition, assuming (in their notation) $C=\mathbb{Q}$ which is all we need. First, note that the assumption that $E$ becomes isogenous over $L$ to a product of elliptic curves is not necessary and indeed not used in the proof, all that is used is that $\operatorname{End}_{L}(A)^{0}$ is a quaternion algebra. Moreover, there is a possible source of confusion in the formulation in that one has to allow the "quadratic extension" mentioned in cases (i), $n=2$ and (ii) to be $\mathbb{Q} \times \mathbb{Q}$ (e.g. $L / F$ quadratic, $A=E_{1} \times E_{2}$ where $E_{1}$ and $E_{2}$ do not have $\mathrm{CM}$ and are non-isomorphic quadratic twists of each other with respect to $L / F)$.

Thus we see that $\rho_{A, \ell}$ is a direct sum of two two-dimensional representations $\rho_{A, \lambda_{1}}$ and $\rho_{A, \lambda_{2}}$ and that there is a finite order character $\epsilon$ such that $\rho_{A, \lambda_{1}} \cong \rho_{A, \lambda_{2}} \otimes$ $\epsilon$. Since $\operatorname{End}_{L}(A)^{0}$ is a quaternion algebra, $\rho_{A, \lambda_{1}}$ and $\rho_{A, \lambda_{2}}$ become isomorphic when restricted to $L$ and are irreducible after restriction to any finite extension of $F$. Thus $\rho_{A, \lambda_{1}}=\rho_{A, \lambda_{2}} \otimes \epsilon$ for some character $\epsilon$ of $\operatorname{Gal}(L / F)$. Note that we saw this in Proposition 20 when $\operatorname{End}_{F}(A)^{0}$ is an imaginary quadratic field, and that $\operatorname{det} \rho_{A, \lambda_{1}}=\epsilon \chi_{\ell}$. 
Proposition 26. Let $A$ be an abelian surface of Galois type $\boldsymbol{E}\left[C_{n}\right]$ for $n=1,3,4,6$ or $\boldsymbol{E}\left[C_{2}, \mathbb{C}\right]$ and assume that $F$ is totally real. Then $A$ satisfies the Sato-Tate conjecture.

Proof. Pick a finite place $\lambda$ of $K=\operatorname{End}_{F}(A)^{0}$ (which is always imaginary quadratic by assumption; cf. [FKRS, Proposition 4.7]) and consider the two-dimensional representation $\rho_{A, \lambda}$ which is a direct summand of $\rho_{A, \ell}$. We claim that $G_{\ell}^{1, Z a r}(A)$ is isomorphic to the Zariski closure $G_{\lambda}^{1, Z a r}$ of $\rho_{A, \lambda}\left(G_{F}^{1}\right)$. This follows since $\rho_{A, \ell}=$ $\rho_{A, \lambda} \oplus \rho_{A, \bar{\lambda}}$ and after restriction to $G_{F}^{1}, \rho_{A, \bar{\lambda}} \cong \rho_{A, \lambda} \otimes \operatorname{det}\left(\rho_{A, \lambda}\right)^{-1}$.

Consider the representation $r_{k, w}$ of $S T_{A}$. $-1 \in S T_{A}$ acts by $(-1)^{k}$ and hence we may define an irreducible algebraic representation $R_{k, w}$ of $G_{\ell}^{Z a r}$ such that

$$
L^{S}\left(s, r_{k, w}, A\right)=L^{S}\left(s+k / 2, R_{k, w} \circ \rho_{A, \lambda}\right) .
$$

The weakly compatible system $\left(R_{k, w} \circ \rho_{A, \lambda}\right)_{\lambda}$ is irreducible, totally odd, essentially self-dual and also regular (the latter is easily seen by restricting to $\operatorname{Gal}(\bar{F} / L)$ ). Hence we deduce invertibility of $L^{S}\left(s+k / 2, R_{k, w} \circ \rho_{A, \ell}\right)$ in the case $k \geq 1$ in the usual way from [BLGGT, Theorem 5.4.1] and Brauer's theorem. When $k=0$ and $w \neq 0$ invertibility follows from the fact that $R_{k, w} \circ \rho_{A, \ell}$ is a non-trivial character of $\operatorname{Gal}(L / F)$.

Proposition 27. Let $A$ be an abelian surface of Galois type $\boldsymbol{E}\left[C_{2}, \mathbb{R} \times \mathbb{R}\right]$ and assume that $F$ is totally real. Then A satisfies the Sato-Tate conjecture.

Proof. Recall that $S T_{A}=J\left(E_{1}\right) \cong \mathrm{SU}(2) \times C_{2}$ and the irreducible representations are of the form $r_{k, w}=S y m^{k} S t \otimes \chi_{w}, k$ integer $\geq 0$ and $w \in 0,1$, with $w=0$ corresponding to the trivial character of $C_{2}$. Moreover $\rho_{A, \ell}=\rho_{A, \lambda} \oplus \rho_{A, \bar{\lambda}}$ where $\lambda$ is a homomorphism $\operatorname{End}_{F}(A)^{0} \rightarrow \overline{\mathbb{Q}}_{\ell}$ and $\bar{\lambda}$ is the other one. We have $\rho_{A, \lambda} \cong$ $\rho_{A, \bar{\lambda}} \otimes \epsilon$ where $\epsilon$ is the non-trivial character $\operatorname{Gal}(L / F) \rightarrow\{ \pm 1\}$. This presentation corresponds to conjugating $J\left(E_{1}\right)$ inside $\mathrm{GL}_{4}(\mathbb{C})$ by e.g. the matrix

$$
\left(\begin{array}{cc}
I & J_{2} \\
I & -J_{2}
\end{array}\right)
$$

this sends $\mathrm{SU}(2)$, embedded as

$$
A \mapsto\left(\begin{array}{cc}
A & \\
& \bar{A}
\end{array}\right),
$$

to SU2 embedded as

$$
A \mapsto\left(\begin{array}{cc}
A & \\
& A
\end{array}\right)
$$

and $J$ gets sent to $\left(\begin{array}{cc}I & \\ & -I\end{array}\right)$. From this we see that the conjugacy classes $s_{v}$ are independent of $\ell$ as they are determined by the trace of $F r o b_{v}$ in the weakly compatible system $\rho_{a, \lambda}$ in the case that $\operatorname{End}_{F}(A)^{0}$ is a field, and by the traces of Frob $v$ in the two weakly compatible systems $\rho_{a, \lambda_{1}}$ and $\rho_{a, \lambda_{2}}$ in the case where $\operatorname{End}_{F}(A)^{0} \cong \mathbb{Q} \times \mathbb{Q}$, where $\lambda_{i}$ is the homomorphism $\mathbb{Q} \times \mathbb{Q} \rightarrow \overline{\mathbb{Q}}_{\ell}$ corresponding to projection to the $i$ th factor. Now pick an extension $R_{k, w}$ of $r_{k, w}$ as usual; then the $R_{k, w} \circ \rho_{A, \ell}$ form an essentially self-dual, odd, regular and irreducible weakly compatible system (where compatibility follows from independence of the $s_{v}$ ) to which we may apply BLGGT, Theorem 5.4.1] when $k \geq 1$ and deduce invertibility as usual, and for $k=0, w=1$ we proceed as usual as well. 
Remark 28. Proposition 27 could also have been proven in similar fashion to Proposition 26 (and vice versa). The proof given rests on the $\ell$-independence of the $s_{v}$ which is unknown in any significant generality, unlike the $\ell$-independence of $S T_{A}$ (which is of course a prerequisite).

4.6. Galois types $\mathbf{E}\left[D_{n}\right], n=2,3,4,6$. Here $\mathbf{E}\left[D_{n}\right]$ corresponds to $J\left(E_{n}\right), n=$ $2,3,4,6$, where the groups $J\left(E_{n}\right)$ are defined as $\left\langle E_{n}, J\right\rangle$. Note that $J$ commutes with $\mathrm{SU}(2)$ and that $J e^{\pi i / n} J=e^{-\pi i / n}$. Using the notation of the previous subsection we see that $r_{k, w}^{J} \cong r_{k,-w}$. Thus $r_{k, w}$ extends if and only if $w=0$ or $n$; we get irreducible representations $R_{k, 0, \epsilon}, R_{k, n, \epsilon}\left(k \in \mathbb{Z}_{\geq 0}, \epsilon \in\{0,1\}, k \equiv 0\right.$ resp. $n$ modulo 2) and $R_{k, w}=\operatorname{Ind}_{E_{n}}^{J\left(E_{n}\right)} r_{k, w}\left(k \in \mathbb{Z}_{\geq 0}, w \in(\mathbb{Z} / 2 n \mathbb{Z}) \backslash\{0, n\}, k \equiv w(2)\right)$. In the case $n=2$ we may also describe the representations of $J\left(E_{2}\right)$ using the subgroup $J\left(E_{1}\right)$ instead of $E_{2}$; they divide into induced and extended as before.

Let $A / F$ be an abelian surface of Galois type $\mathbf{E}\left[D_{n}\right]$ for $n \in\{2,3,4,6\}$. By [FKRS, Proposition 4.7] there is a quadratic extension $F^{\prime} / F$ such that $A / F^{\prime}$ has type $\mathbf{E}\left[C_{n}\right]$ if $n \in\{3,4,6\}$ and $\mathbf{E}\left[C_{2}, \mathbb{C}\right]$ or $\mathbf{E}\left[C_{2}, \mathbb{R} \times \mathbb{R}\right]$ if $n=2$ (in the latter case $F^{\prime}$ is not unique, but any choice will do).

Proposition 29. Let $A / F$ be an abelian surface of Galois type $\boldsymbol{E}\left[D_{n}\right]$ for $n \in$ $\{2,3,4,6\}$ and assume that $F^{\prime}$ is a totally real field. Then the Sato-Tate conjecture holds.

Proof. The proof follows the same lines as the proof of Proposition 24, If $R$ is an irreducible representation of $S T_{A}$ of dimension $\geq 2$, then if $R$ is induced from $S T_{A_{F^{\prime}}}$ one reduces directly to the proof of Proposition 26 for the invertibility of the corresponding $L$-function, and if $R$ is extended from $S T_{A_{F^{\prime}}}$ one argues as in the proof of Proposition 24, using the proof of Proposition 26] or Proposition 27 in place of that of Proposition 22. Finally if $\operatorname{dim} R=1$ we use surjectivity onto the component group as usual.

\section{Appendix A. By Francesc Fité}

Note that Propositions 24 and 29 need the assumption that a certain quadratic extension $F^{\prime}$ of $F$ is such that $F^{\prime}$ is a totally real field.

This is not always the case for the examples in [FKRS, Table 11], and one may wonder about the existence of obstructions imposed by the arithmetic of certain Galois types to this hypothesis ever being satisfied.

The purpose of this section is to show that this is not the case. We will present explicit examples of abelian surfaces satisfying the hypothesis of Proposition 24 and Proposition 29 (in each of their cases), showing that their statements are indeed never vacuous.

Examples meeting the assumptions of Proposition 24 can be constructed in the following way. Consider an elliptic curve $E / F^{\prime}$, where $F^{\prime}$ is a real quadratic field, whose $j$-invariant $j(E)$ lies in $F^{\prime} \backslash \mathbb{Q}$. If $\sigma$ denotes the non-trivial endomorphism of $F^{\prime} / \mathbb{Q}$, then the abelian surface given by descending $E \times E^{\sigma}$ to $\mathbb{Q}$ satisfies the hypothesis of Proposition 24, provided that $E$ and $E^{\sigma}$ are not $\overline{\mathbb{Q}}$-isogenous. An explicit example of this construction is given by the Jacobian of the following genus 2 curve:

$$
C: y^{2}=x(x-1)\left(x^{4}+2 x^{3}-6 x+1\right) .
$$


The curve $C$ has a non-hyperelliptic involution

$$
\alpha(x, y)=\left(\frac{x-1}{x+1}, \frac{-\sqrt{2} y}{(x+1)^{3}}\right) .
$$

Let $F^{\prime}=\mathbb{Q}(\sqrt{2})$ and let $E$ denote the quotient curve $C /\langle\alpha\rangle$. The elliptic curve $E$ may be given by the affine equation

$$
E: y^{2}=x^{3}+\frac{19 \sqrt{2}+22}{2} x^{2}-\frac{18 \sqrt{2}+23}{2} x+\frac{7 \sqrt{2}+10}{4} .
$$

It follows that $\operatorname{Jac}(C)$ is isogenous to $E \times E^{\sigma}$ over $F^{\prime}$. Since $E$ has $j$-invariant $j(E)=\frac{1}{5}(2142720 \sqrt{2}+3039232)$, it does not have complex multiplication. One may now apply [FKRS, Lemma 4.12] (choosing $p=7$, for example) to deduce that $E$ and $E^{\sigma}$ are not $\overline{\mathbb{Q}}$-isogenous. It follows that $L=F^{\prime}$ and that the Galois type of $\operatorname{Jac}(C)$ is $\mathbf{B}\left[C_{2}\right]$.

There are also examples of absolutely simple abelian surfaces of type $\mathbf{B}\left[C_{2}\right]$ with $L$ totally real. As Benjamin Smith pointed out to us, one may find such examples inside the family

$$
C_{t}: y^{2}=x^{5}-5 x^{3}+5 x+t,
$$

for some choices of $t \in \mathbb{Q}$. In TTV91] it is shown that $\operatorname{End}_{\overline{\mathbb{Q}}}\left(\operatorname{Jac}\left(C_{t}\right)\right)^{0}$ contains $\mathbb{Q}(\sqrt{5})$ with this real multiplication being only defined over $\mathbb{Q}(\sqrt{5})$. For particular choices of $t \in \mathbb{Q}$, one can argue that $\operatorname{Jac}\left(C_{t}\right)$ has no further endomorphisms. To do this, one can use the factorization of a few local factors to show that $\operatorname{Jac}\left(C_{t}\right)$ is absolutely simple, and that it does not have neither complex nor quaternionic multiplication. For example, the choice $t=1$ works, by looking at the local factors at $p=11$ and $p=19$.

Regarding Proposition 29, the case $\mathbf{E}\left[D_{2}\right]$ is clear: since any biquadratic extension of $\mathbb{Q}$ contains at least one real quadratic extension, Proposition 29 applies to any abelian surface over $\mathbb{Q}$ of type $\mathbf{E}\left[D_{2}\right]$ (for example, the one given in [FKRS, Table 11]).

For the case $\mathbf{E}\left[D_{4}\right]$, we will use the parametrizations of genus 2 curves with automorphism group isomorphic to the dihedral group of 8 elements $D_{4}$, given in CQ07. The choice of parameters

$$
s=1, \quad u=1 / 2, \quad v=2, \quad z=1,
$$

in [CQ07, Prop. 4.3] yields a curve isomorphic to

$$
C: y^{2}=15 x^{6}-48 x^{5}-6 x^{4}-12 x^{2}+192 x+120 .
$$

It is well known that in this case one has $\operatorname{Jac}(C) \sim_{\tilde{L}} E^{2}$, where $E$ is an elliptic curve defined over the field $\tilde{L}$ of definition of the automorphisms of $C$. By [Car04, §2], the $j$-invariant of $E$ has two possibilities

$$
j(E)=\frac{2^{6}(3 \pm 5 \sqrt{2})^{3}}{(1 \pm \sqrt{2})(1 \mp \sqrt{2})^{2}},
$$


from which one can deduce that $E$ does not have complex multiplication. This implies that $L=\tilde{L}$. We may now use CQ07, Prop. 3.3] to deduce that

$$
L=\mathbb{Q}(\sqrt{2}, \sqrt{1-1 / \sqrt{3}})) .
$$

The Galois type of $\operatorname{Jac}(C)$ is $\mathbf{E}\left[D_{4}\right]$, provided that $\operatorname{Gal}(L / \mathbb{Q}) \simeq D_{4}$. To conclude, note that the three quadratic subextensions $\mathbb{Q}(\sqrt{3}), \mathbb{Q}(\sqrt{2})$, and $\mathbb{Q}(\sqrt{6})$ of $L / \mathbb{Q}$ are real.

Analogously, for the case $\mathbf{E}\left[D_{6}\right]$, we will use the parametrizations of genus 2 curves with automorphism group isomorphic to the dihedral group of 12 elements $D_{6}$, also given in CQ07. Choosing now parameters

$$
s=1, \quad u=2, \quad v=7 / 3, \quad z=1,
$$

in [CQ07, Prop. 4.9], we obtain a curve isomorphic to

$$
C: y^{2}=x^{5}+12 x^{4}-2124 x^{3}+7992 x^{2}+329184 x-38880 .
$$

As in the previous example, one has $\operatorname{Jac}(C) \sim_{\tilde{L}} E^{2}$, where $E$ is an elliptic curve defined over the field $\tilde{L}$ of definition of the automorphisms of $C$. By [Car04, §2], the $j$-invariant of $E$ has two possibilities

$$
j(E)=\frac{2^{8} 3^{3}(2 \pm 5 \sqrt{2})^{3}(\mp \sqrt{2})}{(1 \pm 2 \sqrt{2})(1 \mp 2 \sqrt{2})^{3}},
$$

from which one can deduce that $E$ does not have complex multiplication. Again this implies that $L=\tilde{L}$, which may be computed by means of CQ07, Prop. 3.5]. Indeed, one finds that $\tilde{L}$ is the compositum of $\mathbb{Q}(\sqrt{2})$ and the splitting field of

$$
x^{3}-\frac{3}{2} x-\frac{1}{4} .
$$

Since $\operatorname{Gal}(L / \mathbb{Q}) \simeq D_{6}$, we have that the Galois type of $\operatorname{Jac}(C)$ is $\mathbf{E}\left[D_{6}\right]$. The three quadratic subextensions $\mathbb{Q}(\sqrt{2}), \mathbb{Q}(\sqrt{42})$, and $\mathbb{Q}(\sqrt{21})$ of $L / \mathbb{Q}$ are all real. Taking $F^{\prime}=\mathbb{Q}(\sqrt{21})$, we see that $\operatorname{Jac}(C) / F^{\prime}$ has Galois type $\mathbf{E}\left[C_{6}\right]$.

For the case $\mathbf{E}\left[D_{3}\right]$, consider $\operatorname{Jac}(C) / F$, where $C$ is as in $(\mathrm{A} .2)$ and $F=\mathbb{Q}(\sqrt{2})$. The Galois type of $\operatorname{Jac}(C) / F$ is $\mathbf{E}\left[D_{3}\right]$ and the Galois type of $\operatorname{Jac}(C) / F^{\prime}$, with $F^{\prime}=\mathbb{Q}(\sqrt{2}, \sqrt{21})$, is $\mathbf{E}\left[C_{3}\right]$.

\section{ACKNOWLEDGEMENTS}

C. Johansson wishes to thank Toby Gee for useful remarks on an earlier draft of this paper, Kiran Kedlaya and Jean-Pierre Serre for highlighting the issue of the two different definitions of a Sato-Tate group in the potentially abelian case, Andrew Wiles for a useful conversation, and the anonymous referee for useful comments and corrections. Moreover thanks are also due to Kedlaya, Benjamin Smith and Francesc Fité for discussions relating to the Appendix. This research was supported by EPSRC Grant EP/J009458/1.

F. Fité wishes to thank Andrew Sutherland for assistance in several computations, as well as for kindly providing the curve of (A.1), which was found using the techniques of [KS08]. 


\section{REFERENCES}

[AC] James Arthur and Laurent Clozel, Simple algebras, base change, and the advanced theory of the trace formula, Annals of Mathematics Studies, vol. 120, Princeton University Press, Princeton, NJ, 1989. MR 1007299

[AT] Emil Artin and John Tate, Class field theory, 2nd ed., Advanced Book Classics, Addison-Wesley Publishing Company, Advanced Book Program, Redwood City, CA, 1990. MR 1043169

[BK] Grzegorz Banaszak and Kiran S. Kedlaya, An algebraic Sato-Tate group and Sato-Tate conjecture, Indiana Univ. Math. J. 64 (2015), no. 1, 245-274, DOI 10.1512/iumj.2015.64.5438. MR3320526

[BLGG] Thomas Barnet-Lamb, Toby Gee, and David Geraghty, The Sato-Tate conjecture for Hilbert modular forms, J. Amer. Math. Soc. 24 (2011), no. 2, 411-469, DOI 10.1090/S0894-0347-2010-00689-3. MR2748398

[BLGGT] Thomas Barnet-Lamb, Toby Gee, David Geraghty, and Richard Taylor, Potential automorphy and change of weight, Ann. of Math. (2) 179 (2014), no. 2, 501-609, DOI 10.4007/annals.2014.179.2.3. MR.3152941

[BGHT] Tom Barnet-Lamb, David Geraghty, Michael Harris, and Richard Taylor, A family of Calabi-Yau varieties and potential automorphy II, Publ. Res. Inst. Math. Sci. 47 (2011), no. 1, 29-98, DOI 10.2977/PRIMS/31. MR.2827723

[Car04] Gabriel Cardona, $\mathbb{Q}$-curves and abelian varieties of $\mathrm{GL}_{2}$-type from dihedral genus 2 curves, Modular curves and abelian varieties, Progr. Math., vol. 224, Birkhäuser, Basel, 2004, pp. 45-52. MR2058641

[CQ07] Gabriel Cardona and Jordi Quer, Curves of genus 2 with group of automorphisms isomorphic to $D_{8}$ or $D_{12}$, Trans. Amer. Math. Soc. 359 (2007), no. 6, 2831-2849 (electronic), DOI 10.1090/S0002-9947-07-04111-6. MR2286059

[Far] L. Fargues, Motives and automorphic forms: The (potentially) abelian case, available at http://www.math.jussieu.fr/ ${ }^{\sim}$ fargues/Motifs_abeliens.pdf.

[FKRS] Francesc Fité, Kiran S. Kedlaya, Víctor Rotger, and Andrew V. Sutherland, SatoTate distributions and Galois endomorphism modules in genus 2, Compos. Math. 148 (2012), no. 5, 1390-1442, DOI 10.1112/S0010437X12000279. MR2982436

[FS] Francesc Fité and Andrew Sutherland, Sato-Tate distributions of twists of $y^{2}=x^{5}-x$ and $y^{2}=x^{6}+1$, preprint, available at http://arxiv.org/abs/1203.1476.

[Har] Michael Harris, Potential automorphy of odd-dimensional symmetric powers of elliptic curves and applications, Algebra, arithmetic, and geometry: in honor of Yu. I. Manin. Vol. II, Progr. Math., vol. 270, Birkhäuser Boston, Inc., Boston, MA, 2009, pp. 1-21, DOI 10.1007/978-0-8176-4747-6_1. MR2641185

[HSBT] Michael Harris, Nick Shepherd-Barron, and Richard Taylor, A family of Calabi-Yau varieties and potential automorphy, Ann. of Math. (2) 171 (2010), no. 2, 779-813, DOI 10.4007/annals.2010.171.779. MR.2630056

[KS08] Kiran S. Kedlaya and Andrew V. Sutherland, Computing L-series of hyperelliptic curves, Algorithmic number theory, Lecture Notes in Comput. Sci., vol. 5011, Springer, Berlin, 2008, pp. 312-326, DOI 10.1007/978-3-540-79456-1_21. MR2467855

[NSW] Jürgen Neukirch, Alexander Schmidt, and Kay Wingberg, Cohomology of number fields, Grundlehren der Mathematischen Wissenschaften [Fundamental Principles of Mathematical Sciences], vol. 323, Springer-Verlag, Berlin, 2000. MR 1737196

[Rib] Kenneth A. Ribet, Abelian varieties over $\mathbf{Q}$ and modular forms, Algebra and topology 1992 (Taejŏn), Korea Adv. Inst. Sci. Tech., Taejŏn, 1992, pp. 53-79. MR1212980

[Ser1] Jean-Pierre Serre, Abelian l-adic representations and elliptic curves, McGill University lecture notes written with the collaboration of Willem Kuyk and John Labute, W. A. Benjamin, Inc., New York-Amsterdam, 1968. MR0263823

[Ser2] Jean-Pierre Serre, Lectures on $N_{X}(p)$, Chapman \& Hall/CRC Research Notes in Mathematics, vol. 11, CRC Press, Boca Raton, FL, 2012. MR2920749

[Tat] J. Tate, Number theoretic background, Automorphic forms, representations and $L$ functions (Proc. Sympos. Pure Math., Oregon State Univ., Corvallis, Ore., 1977), Proc. Sympos. Pure Math., XXXIII, Amer. Math. Soc., Providence, R.I., 1979, pp. 3-26. MR 546607 
[TTV91] Walter Tautz, Jaap Top, and Alain Verberkmoes, Explicit hyperelliptic curves with real multiplication and permutation polynomials, Canad. J. Math. 43 (1991), no. 5, 1055-1064, DOI 10.4153/CJM-1991-061-x. MR1138583

[Tay] Richard Taylor, Remarks on a conjecture of Fontaine and Mazur, J. Inst. Math. Jussieu 1 (2002), no. 1, 125-143, DOI 10.1017/S1474748002000038. MR.1954941

[Wei] André Weil, Sur la théorie du corps de classes (French), J. Math. Soc. Japan 3 (1951), 1-35. MR 0044569

School of Mathematics, Institute for Advanced Study, Princeton, New Jersey 08540 Current address: Department of Pure Mathematics and Mathematical Statistics, University of Cambridge, Cambridge CB3 0WB, United Kingdom

E-mail address: hcj24@cam.ac.uk

Universitat Politecnica de Catalunya, Edifici Omega, C/Jordi-Girona 1-3, E-08034 BARCELONA, SPAIN

E-mail address: francesc.fite@gmail.com 